Philosophie ANTIQUE
Philosophie antique

Problèmes, Renaissances, Usages

13 | 2013

Analogie et connaissance

\title{
Les peintres d'Empédocle (DK 31 B 23)
}

Enjeux et portée d'une analogie préplatonicienne

\section{Leopoldo Iribarren}

\section{(2) OpenEdition}

Journals

Édition électronique

URL : https://journals.openedition.org/philosant/902

DOI : $10.4000 /$ philosant.902

ISSN : 2648-2789

Éditeur

Éditions Vrin

\section{Édition imprimée}

Date de publication : 1 novembre 2013

Pagination : 83-115

ISBN : 978-2-7574-0591-8

ISSN : 1634-4561

\section{Référence électronique}

Leopoldo Iribarren, « Les peintres d'Empédocle (DK 31 B 23) », Philosophie antique [En ligne], 13 | 2013, mis en ligne le 01 novembre 2018, consulté le 02 décembre 2022. URL : http://

journals.openedition.org/philosant/902 ; DOI : https://doi.org/10.4000/philosant.902

\section{(c) (i) (9)}

Creative Commons - Attribution - Pas d'Utilisation Commerciale - Pas de Modification 4.0 International - CC BY-NC-ND 4.0

https://creativecommons.org/licenses/by-nc-nd/4.0/ 


\title{
LES PEINTRES D'EMPÉDOCLE (DK 31 B23) ENJEUX ET PORTÉE D'UNE ANALOGIE PRÉPLATONICIENNE*
}

\author{
Leopoldo IRIBARREN
}

Centre de recherches sur la pensée antique, CNRS, Paris

RÉSUMÉ. Cet article est consacré au fragment B23 d'Empédocle, où l'auteur met en relation la génération des espèces vivantes à partir des quatre éléments avec l'art pictural dont les productions résultent des divers mélanges d'un nombre limité de pigments. La première partie aborde la dimension heuristique de l'analogie dans le cadre du récit cosmologique, notamment la question de la correspondance entre comparans et comparandum. À l'intérieur de cette relation, un problème grammatical et théorique retient mon attention : alors que le sujet et les verbes du fragment sont au pluriel, les procédés des peintres sont exprimés par des participes au duel. Ces duels ont souvent été interprétés comme des allusions aux deux puissances cosmiques d'Empédocle, l'Amour et la Haine. Prenant appui sur l'interprétation récente du cycle cosmique donnée par Oliver Primavesi, selon laquelle même dans la période où la Haine domine, l'Amour reste le principe de production des espèces mortelles, je propose d'interpréter les duels comme une allusion aux mains de Cypris, l'une des personnifications du pouvoir démiurgique de l'Amour. En ce sens, la lecture que je propose ici de l'analogie s'avère compatible avec les deux courants interprétatifs principaux de la zoogonie d'Empédocle (unique/double). Dans la deuxième partie de l'article, j'analyse l'écart réflexif que l'analogie des peintres introduit par rapport à deux formes de discours contemporaines d'Empédocle qui thématisent la question de la représentation : d'une part, la tradition lyrique, notamment Simonide et Pindare; de l'autre, le poème philosophique de Parménide.

\footnotetext{
*. Ce texte fait partie des recherches que j'ai pu mener grâce au soutien de la Fondation Maison des Sciences de l'Homme et de la Commission Européenne (COFUND Marie Curie), au sein du labex RESMED. Que ces institutions soient remerciées de leur accueil. Le travail que je présente ici a bénéficié d'échanges avec Jean-Claude Picot, dont la connaissance d'Empédocle va de pair avec sa générosité. Une fois encore, les remarques d'André Laks ont contribué à préciser mes idées. La traduction des textes grecs cités est mienne. Sans autre indication, je rapporte les fragments des Présocratiques selon la numérotation de Diels-Kranz.
}

Philosophie antique, $\mathrm{n}^{\circ} 13$ (2013), 83-115 
SUMMARY. This paper focuses on Empedocles' fragment B23, in which the author analogically relates the generation of living species from four elements to the art of painting, whose productions result from the mixing of a limited number of pigments. The first part of the article deals with the heuristic dimension of the analogy in the context of a cosmological poem, more specifically with the correspondence between comparans and comparandum. Within this relationship, a grammatical and theoretical problem concentrates my attention: while the subject and the verbs of the fragment are plurals, the painter's procedures are conveyed in the dual form. These duals have frequently been interpreted as allusions to the two cosmic principles of Empedocles' doctrine, Love and Strife. Building on Oliver Primavesi's recent interpretation of the cosmic cycle, according to which Love is the only principle responsible for the production of living species, even during the period of Strife's domination, I propose to interpret the duals as an allusion to Cypris' hands, a personification of Love's demiurgical powers. As a result, the reading I suggest is compatible with the two main interpretations of Empedocles' zoogony (single/double). In the second part of the paper, I examine the relation that the painters analogy entertains with two contemporary discursive forms that also happen to thematize the question of representation: the lyric tradition, namely Simonides and Pindar, on the one hand, and the philosophical poem of Parmenides, on the other. 
Jedes Existierende ist ein Analogon alles Existierenden; daher erscheint uns das Dasein immer zu gleicher Zeit gesondert und verknüpft. Folgt man der Analogie zu sehr, so fällt alles identisch zusammen; meidet man sie, so zerstreut sich alles ins Unendliche. In beiden Fällen stagniert die Betrachtung, einmal als überlebendig, das andere Mal als getötet.

Goethe, Maximen und Reflexionen, a. a. O., vol. 12, p. 368.

Parmi les diverses analogies et métaphores employées par Empédocle dans son poème physique, celles qui ont trait au thème de l'artisanat sont de loin les plus fréquentes. Qu'il s'agisse d'expliquer des phénomènes naturels, tels la formation des parties du corps et leur physiologie, ou artistiques, comme la composition du poème et sa poétique immanente, la technique fournit des modèles transposables analogiquement aux domaines de réalité qui sont par nature inaccessibles à la perception immédiate ${ }^{1}$. Certes, Empédocle n'est pas le premier penseur à avoir exploité les vertus heuristiques du paradigme artisanal. La nouveauté qu'il introduit dans l'emploi de ce modèle consiste plutôt dans la réunion de ses deux champs d'application traditionnels : le poétologique et le cosmologique. Alors que les traditions épiques et lyriques ont privilégié l'usage de comparaisons techni-

1. Kranz 1938 dresse une liste partielle des modèles techniques que je complète à partir des fragments et des témoignages : le char et le poème (B3), le char et le cheminement de la lune (B46), la lanterne et l'œil (B84), la clepsydre et la respiration (B100), le rebondissement du projectile et la réflexion du soleil sur la lune (B43), la coupe qu'on fait tourner dans l'air et le tournoiement de la voûte céleste (A67), le gnomon et la nuit (B48), la formation des eaux chaudes sous la terre et le serpentin du chauffe-eau (A68). Les procédés techniques de certains métiers sont évoqués à titre analogique: le chimiste (A34), le fondeur (B92), le fromager (B33), le boulanger (B34), le vitrificateur (A51), le potier (B73), le peintre (B23), le charpentier (B87, B96. 4). Des métaphores techniques servent aussi à nommer des organes du corps (B70, B100.8). 
ques pour la description de la composition poétique en tant que telle, les philosophes ioniens les ont mobilisées à des fins de modélisation cosmologique ${ }^{2}$. Héritier aussi bien des formes poétiques archaïques que des premières spéculations philosophiques, Empédocle réunit dans le poème physique les deux champs d'application traditionnels de l'analogie technique. D’une part, son emploi quasi systématique pour illustrer des phénomènes naturels inaccessibles à la perception, telles la respiration (B100) ou la vision (B84), témoigne de l'émergence d'un mode de rationalisation d'hypothèses explicatives et prédictives ${ }^{3}$. D'autre part, les comparaisons techniques chez Empédocle font apparaître, dans leur structure, syntaxe et vocabulaire, des rapports réflexifs avec des traditions poétiques non philosophiques, dont l'impact proprement théorique (et pas seulement formel) sur la doctrine peut s'avérer considérable ${ }^{4}$. Comme je me propose de le montrer ici, la dimension métapoétique d'une comparaison n'est pas moins porteuse de sens que la dimension proprement cognitive que l'on dégage $\mathrm{du}$ tertium comparationis. Mon cas d'étude sera l'analogie des peintres (B23), où Empédocle met en relation la génération des espèces vivantes à partir des quatre éléments avec l'art pictural dont les productions résultent des divers mélanges d'un nombre limité de pigments.

L'emploi de cette analogie dans un contexte cosmologique soulève un certain nombre de questions qui touchent non seulement à la doctrine physique d'Empédocle, mais aussi, dans une perspective plus large, aux débuts historiques d'une théorie de la représentation. Afin de mieux distinguer les enjeux, je diviserai mon analyse en deux parties. La première partie abordera la dimension heuristique de l'analogie des peintres dans le cadre du

2. Pour la poésie archaïque, je renvoie à l'inventaire exhaustif établi par Nünlist 1998, p. 83-134. Dans la philosophie présocratique, on peut signaler chez Anaximandre les comparaisons des phénomènes célestes avec des objets techniques tels une colonne, une roue ou le bec d'un soufflet (DK 12 A11, A21, B2, B4), chez Anaximène le mouvement des étoiles autour de la Terre est comparé à celui d'un bonnet de feutre sur la tête (DK 13 A7). Un cas particulièrement intéressant dans le contexte présocratique est celui de Phérécyde, où la Terre et l'Océan sont créés à partir d'une broderie cosmique (DK 7 B2). Chez Parménide le paradigme technique apparaît dans le proème (DK 28 B1) avec la métaphore du char, dont les roues symbolisent l'agencement dynamique des deux parties du poème révélé par la Déesse. De même, les portes du Jour et de la Nuit annoncent la structure duale de la vision du monde élaborée du point de vue des mortels.

3. Cf. Diller 1932; Kranz 1938; Snell 1953, p. 213-218; Lloyd 1966, p. 304-383; O’Brien 1970.

4. Voir Bollack 1965, p. 295-310. Plus récemment, Rashed 2007 a mis en évidence le rapport réflexif qu'Empédocle établit dans le fragment B84 entre l'assemblage de l'œil par Aphrodite et la fabrication du radeau d'Ulysse (Od. V, 247-259). Au-delà des similitudes formelles et lexicales entre les deux passages, le nostos d'Ulysse apparaît comme le thème qui, de manière réflexive, explicite la dimension cosmologique de l'œil : le chef-d'œuvre d'Aphrodite est en même temps une anticipation du retour de Sphairos. 
récit cosmologique. Il s'agira notamment d'examiner à nouveaux frais la question de la correspondance entre comparans et comparandum. Si les pigments ( $\phi \dot{\alpha} p \mu \alpha \kappa \alpha, v .3)$ dont se servent les peintres renvoient aux quatre éléments constitutifs de la nature, comme cela ne fait pas de doute, à quoi correspondent les peintres dont les procédés sont exprimés par des participes au duel ( $\delta \varepsilon \delta \alpha \tilde{\omega} \tau \varepsilon ; \mu \varepsilon i \xi \alpha \nu \tau \varepsilon ; \kappa \tau i \zeta o v \tau \varepsilon$, v. $2,4,6)$ alors que le sujet et les verbes sont au pluriel ? Qualifiés par les éditeurs modernes de trait stylistique sans répercussion sémantique ${ }^{5}$, ces duels ont fait l'objet de réévaluations récentes qui les ont investis d'une signification théorique. Ils renverraient, selon les spécialistes, aux deux puissances cosmiques de la doctrine d'Empédocle, l'Amour et la Haine, avec pour conséquence l'attribution d'un rôle démiurgique à cette dernière ${ }^{6}$. Si, comme le soutient l'interprétation traditionnelle du cycle empédocléen, une zoogonie sous la Haine fait vraisemblablement partie de la cosmologie, l'assimilation de cette zoogonie à une forme de démiurgie dont la Haine serait l'agent (représentée sous les traits d'un peintre aux côtés de l'Amour dans le fragment B23) est quant à elle problématique. Prenant appui sur l'interprétation récente donnée par Oliver Primavesi du cycle cosmique, selon laquelle même dans la période où la Haine domine, c'est l'Amour qui reste le principe de la production des espèces mortelles ${ }^{7}$, je propose d'interpréter les duels comme une allusion aux mains de Cypris, l'une des personnifications du pouvoir démiurgique de l'Amour. En ce sens, la lecture que je présente ici de l'analogie des peintres est compatible avec les deux schémas zoogoniques qui, avec des variantes plus ou moins importantes, ont été proposés par les interprètes ${ }^{8}$. La deuxième partie de cette étude sera consacrée à l'analyse du rapport qu'Empédocle construit dans ce même fragment B23 entre peinture, poésie et monde phénoménal. Au-delà de l'utilité heuristique première que présente la comparaison des peintres - circonscrite au processus de la génération dans la doctrine physique -, l'analogie met en relief un rapport spécifique entre les sphères cosmologique et symbolique (poétique et picturale) par lequel Empédocle se démarque à la fois de la tradition lyrique et de la spéculation philosophique en vers. L'emploi de l'analogie des peintres produit effectivement chez Empédocle un double

5. Bollack 1969, p. 122 ; Gallavotti 1975, p. 211 ; Wright 1981, p. 179-180.

6. Trépanier 2003 ; Sedley 2007, p. 59.

7. Mansfeld et Primavesi 2011, p. 403.

8. Pour le schéma de la double zoogonie, voir notamment: Panzerbieter 1844; Zeller 1920, p. 971 ; Diels 1901, p. 112 ; Bignone 1916, p. 545-598 ; Wright 1981, p. 41 ; Graham 1988 ; Inwood 2001, p. 41-43; Martin et Primavesi 1999, p. 75-82; Trépanier 2003; Primavesi 2008; Mansfeld et Primavesi 2011, p. 396-404. Pour le schéma de la zoogonie unique : von Arnim 1902; Bollack 1965, p. 95-124; Hölscher 1965; Solmsen 1965 ; Long 1974. 
écart réflexif par rapport aux visées propres de deux formes de discours qui lui sont contemporaines: d'une part, la tradition lyrique, notamment Simonide et Pindare, qui définit le statut des formes symboliques de la parole et de l'image en fonction des pratiques commémoratives; de l'autre, le poème philosophique de Parménide, qui soulève la question de la ressemblance entre l'objet même du discours (l'étant) et l'image qu'il construit de cet objet (la sphère). L'analogie des peintres absorbe simultanément deux problématiques distinctes mais contemporaines travaillées respectivement par deux courants de la poésie archaïque : la relation entre formes symboliques et pratiques sociales instituées, d'une part, la fonction de l'image dans un discours qui se donne pour objet la totalité du réel, de l'autre.

\section{I}

\section{L'analogie des peintres dans son contexte cosmologique}

Simplicius est le seul doxographe à nous avoir transmis intégralement le fragment B23. Ses indications sur la localisation du passage le situent au premier livre du Peri physeos, à la suite de B21 dont il serait un éclaircissement. Le lien entre les deux passages est souligné par un commentaire du doxographe : « et il donnait même un exemple qui montre clairement que les différences naissent des mêmes choses (scil. les premiers principes) 》

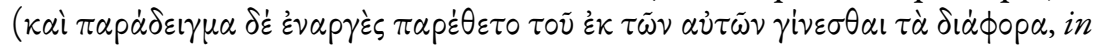
Phys. 159, 27-28). Je rapporte puis traduis les deux fragments dans l'ordre où Simplicius les transmet (in Phys. 159, 13-26 et 160, 1-11):

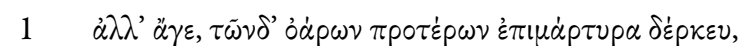

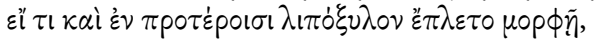

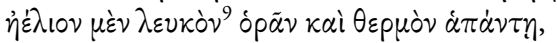

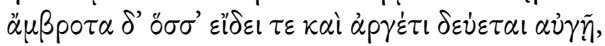

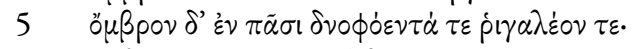

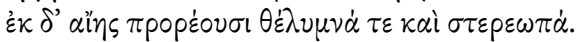

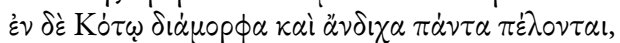

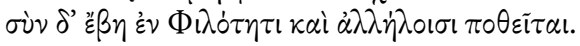

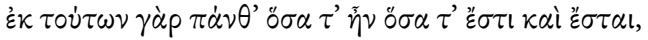

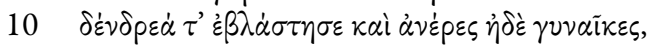

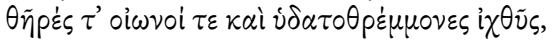

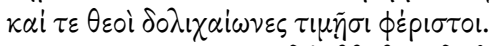
$\alpha \dot{\nu} \tau \dot{\alpha} \gamma \dot{\alpha} \rho \tilde{\varepsilon} \sigma \tau \tau \nu \tau \alpha \tilde{\nu} \tau \alpha, \delta \iota^{\prime} \dot{\alpha} \lambda \lambda \dot{\lambda} \lambda \omega \nu \nu \dot{\varepsilon} \theta \dot{\varepsilon} \sigma \nu \tau \alpha$

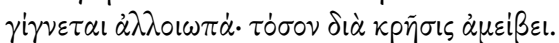

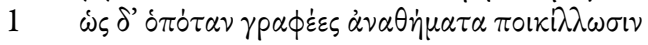

9. Les manuscrits de Simplicius ne fournissent pas $\lambda$ عuкóv. Cette leçon, qui se trouve chez Aristote, a été préférée par Kranz dans ses éditions de Die Fragmente der Vorsokratiker, à partir de 1934 . 


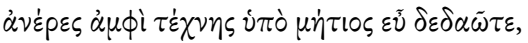

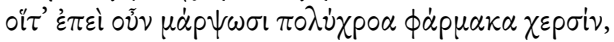

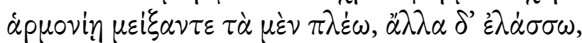

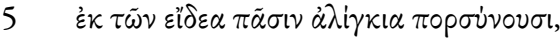

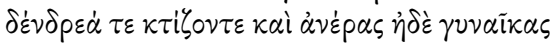

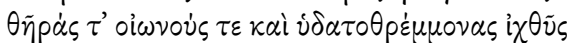

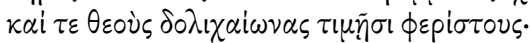

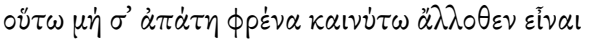

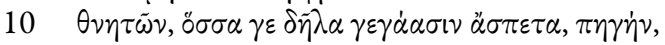

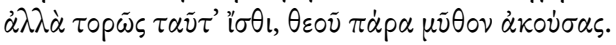

1 Mais viens, considère ce témoignage de mes confidences précédentes, Pour le cas où, dans mes premières paroles, quelque chose manquerait de forme :

Le soleil, blanc à voir et chaud en tout lieu,

Toutes les choses immortelles baignées de chaleur et de brillante lumière,

5 Et la pluie en tous lieux, sombre et glaciale ;

Et du sol coulent les choses, bien fondées et fermes.

Dans Colère, tous ont leurs formes divisées et se séparent,

Tandis que dans Amour ils se réunissent et se désirent l'un l'autre.

Car c'est d'eux que provient tout ce qui fut, tout ce qui est et qui sera,

10 D'eux les arbres ont germé, les hommes et les femmes,

Les bêtes et les oiseaux, les poissons que nourrit l'eau

Et les dieux longévifs, les premiers en honneurs.

Car ils sont eux-mêmes, mais, courant les uns à travers les autres,

Ils deviennent différents en forme : tel est l'échange que provoque le mélange.

1 Comme quand les peintres enluminent les offrandes,

Hommes bien instruits dans leur art grâce à leur habileté,

Ils saisissent de leurs mains les poudres multicolores,

Les mélangeant harmonieusement, tantôt plus, tantôt moins de parts,

5 À partir de quoi ils composent des figures semblables à toutes les choses,

Créant les arbres, les hommes et les femmes,

Les bêtes et les oiseaux, les poissons que nourrit l'eau

Et les dieux longévifs, les premiers en honneurs :

Ainsi, que ta pensée ne succombe pas à l'erreur que c'est d'ailleurs

10 Que provient la source des choses mortelles qui, innombrables, sont là, manifestes,

Mais connais-les clairement, ayant écouté le récit d'un dieu.

Outre l'indication de Simplicius, deux arguments plaident en faveur de la continuité immédiate de ces deux passages dans le poème physique. Le premier s'appuie sur la reprise de la séquence de vers qui détaille l'inventaire du monde (B21. 9-12 B23. 5-8). Dans l'hypothèse de la continuité tex- 
tuelle, cette reprise paraît clairement destinée à mettre en évidence le tertium comparationis reliant le comparandum (le monde physique) au comparans (la représentation picturale). En l'occurrence, le passage du petit nombre au multiple dans l'ordre de la nature est éclairé par un procédé analogue dans l'ordre de la technique. Alors que dans le comparandum les diverses espèces «germent» (B21. $10: \beta \lambda \alpha \sigma \tau \dot{\alpha} \nu \omega$ renvoie au champ sémantique de la nature) à partir des éléments, dans le comparans les créatures sont « créées » (B23. $6: k \tau i \zeta \omega$ renvoie à l'agir artisanal). On peut observer des reprises équivalentes, bien que sur une plus faible échelle, dans les deux autres analogies à thème artisanal qui nous sont parvenues du poème physique. Dans celle qui compare l'œil à une lanterne (B84), la reprise des vers 5 et 11 signale l'identité du mouvement des particules de feu qui a lieu, d'une part, à l'intérieur de la lanterne, et de l'autre, dans l'organe de la vision. Dans l'analogie qui met en rapport la respiration avec le fonctionnement d'une clepsydre (B100), l'alternance des mouvements de l'air et de l'eau dans l'artefact est mise en relief par un jeu de variantes entre les vers 15 et 21. Dans ces deux comparaisons, les reprises formulaires ont pour fonction de diriger l'attention vers un tertium comparationis: le processus décrit dans l'objet technique est aussi à l'œuvre dans le monde physique ${ }^{10}$.

Mon deuxième argument en faveur d'une continuité immédiate entre les deux fragments repose sur la mention de la couleur de deux des quatre

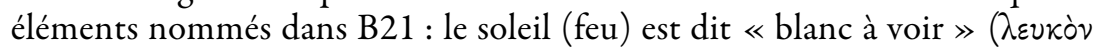

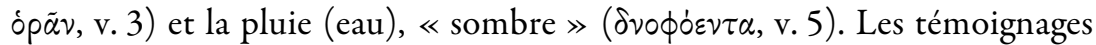
doxographiques coïncident dans l'attribution par Empédocle des couleurs blanc et noir respectivement au feu et à l'eau ; quant aux couleurs des autres éléments, c'est un sujet de débat, mais il n'est pas exclu que le rouge et le jaune aient eu des correspondants élémentaires ${ }^{11}$. De son côté, la peinture ancienne, comme le montre l'évidence textuelle et archéologique, travaillait sur une palette restreinte de quatre couleurs : blanc, noir, rouge et jaune ${ }^{12}$.

10. La reprise d'un mot ou d'un hémistiche pour indiquer le point focal de la comparaison est un procédé poétique fréquent dans l'Iliade. Cf. XVII, 520-523 ; XIX, 356-360 ; XXIII, 222-224.

11. Cf. Théophraste, De sens. 59 (= A69a), qui mentionne seulement le blanc et le noir comme couleurs élémentaires. Aétius, I, 15, 3 (= A92) en mentionne quatre : « il y a quatre couleurs, autant que d'éléments, le blanc, le noir, le rouge, le jaune », sans les attribuer aux éléments correspondants. Comme il a été souvent signalé, il n'est pas improbable que la tradition doxographique ait attribué les quatre couleurs de Démocrite (Théophraste, De sens. 73-75 = DK 68 A135) à Empédocle.

12. Cf. Pline, H. N. XXXV, 50 et 92 ; Cicéron, Brutus, 18. 70 ; Plutarque, De def. or. 436 B-C ; ps. Aristote, De mundo, 396b13. L'évidence archéologique concernant la peinture ancienne est, comme on sait, assez maigre. On peut signaler cependant la célèbre mosaique d'Alexandre à Pompée (copie du III ${ }^{\mathrm{e}}$ siècle d'un original attribué à Philoxène d'Érétrie), réalisée sur la base des quatre couleurs mentionnées. Au sujet des quatre couleurs de la pein- 
Si une correspondance stricte entre les couleurs primaires de la peinture et celles des éléments d'Empédocle ne peut pas être établie de façon certaine, il n'en reste pas moins que l'allusion chromatique en B21 pouvait avoir la fonction d'annoncer le thème de la comparaison des peintres en B23. D'autre part, rien ne s'oppose à ce que $\dot{\omega}^{\prime} \delta^{\prime} \dot{o} \pi \dot{\delta} \tau \alpha \nu$ (B23. 1) relie syntaxiquement la comparaison des peintres au dernier vers de B21, qui insiste sur la génération à partir du mélange. Cette proximité thématique et syntaxique entre les deux passages a conduit la plupart des éditeurs modernes à adopter l'hypothèse de leur continuité immédiate ${ }^{13}$.

Les reconstructions récentes du premier livre de la physique, rendues possibles par l'édition du papyrus de Strasbourg, ont contribué à préciser le contexte dans lequel vraisemblablement figuraient les fragments B21 et B23 ${ }^{14}$. Grâce aux notes stichométriques en marge du papyrus et à l'identification dans son texte de certains passages que l'on connaissait déjà par la tradition indirecte, on a pu en effet reconstituer de façon relativement précise un ensemble de quelques soixante-seize lignes, correspondant aux vers 232-308 du premier livre du poème physique ${ }^{15}$. La relation de l'ensemble $a$ du papyrus avec les fragments B21 et B23 peut être établie à son tour sur la base d'une reprise aux vers $269-272(=a(\mathrm{i}) 8-a$ (ii) 2) des quatre vers formulaires qui postulent la capacité des premiers principes de produire la totalité des espèces vivantes (B21.9-12 B23. 5-8). Les contenus programmatiques de l'ensemble Strasb. a et ceux des fragments B21 et B23 semblent s'inscrire dans un même développement argumentatif, comme cela ressort de la reconstruction du premier livre du poème physique proposée par

ture ancienne, voir Scheibler 1974; Bruno 1977, p. 53-59; Wright 1981, p. 38-39; Ierodiakonou 2004 et 2005 ainsi que l'appendice $n^{\circ} 2$ du volume XXXV de l'édition de Pline réalisée par Croisille 1985, p. 301-303.

13. La continuité entre B21 et B23 a été défendue par Bollack 1969, vol.3, p. 120 et adoptée par Gallavotti 1975, Wright 1981, Inwood 2001 et Sedley 2007, p. 58. Primavesi 2008 insère les sept vers de l'ensemble Strasb. b (reconstruit sur la base de B76) entre B21 et B23. Les raisons de Primavesi ne m'ont pas convaincu (cf. Martin et Primavesi 1999, p. 108). Dans Strasb. $b$, Empédocle attire l'attention du disciple ( $\tau \circ \tilde{\tau} \tau 0 \mu \varepsilon \dot{\nu} . .$.$) sur les espèces animales$ où l'élément terre est prépondérant. Si l'on intercale ce passage entre B21 et B23, comme le suggère Primavesi, la logique du récit serait la suivante : Empédocle insiste sur la génération de toutes les espèces vivantes à partir du mélange des quatre éléments, convoque ensuite les témoignages de la présence de terre dans certaines espèces, et conclut par l'illustration de la génération de toutes les espèces au moyen de l'analogie des peintres. L'intelligibilité du récit gagne, me semble-t-il, avec l'hypothèse de la continuité immédiate entre B21 et B23.

14. Notamment Primavesi 2008. Les reconstructions de Janko 2004 et 2005 ne permettent pas de situer B21 et B23.

15. L'ensemble reconstruit intègre le fragment $\mathrm{B} 17$, dont le papyrus recoupe cinq vers, ainsi que le fragment B20, qu'il recoupe intégralement. L'ensemble commence au vers 263; les vers 262-266 ( $=a(\mathrm{i}) 1-5)$ non mutilés sont aussi préservés par Simplicius (in Phys. 158 $=\mathrm{B} 17.31-35)$. Les sept derniers vers, 302-308 (= $c 1-8)$, correspondent à B20. 
Oliver Primavesi, d'après laquelle les fragments connus s'enchaînent de la manière suivante: B17-ensemble Strasb. a-ensemble Strasb. c-B20-B21ensemble Strasb. b-B23-B26-B35-B27a-B28-B27-B29-B30-B31 ${ }^{16}$.

Une fois établie la position relative qu'occupait l'analogie des peintres dans le premier livre du poème physique, se pose la question de sa portée heuristique. Plus concrètement, quel(s) moment(s) du cycle cosmique Empédocle entendait-il illustrer au moyen de cette analogie ? Avant de tenter d'y répondre, il n'est pas inutile de mettre au clair quelques aspects programmatiques du poème physique dont l'énonciation précédait la comparaison entre le devenir naturel et la peinture. En ce qui concerne le fragment B17 et l'ensemble Strasb. a, le texte n'est pas dénué de difficultés et le sens de certains vers importants est incertain, que ce soit en raison de variantes et de corruptions textuelles, ou du caractère ambigu et métaphorique de certains termes. Malgré ces difficultés, le passage laisse apparaître de manière suffisamment claire le contexte général à la suite duquel devait s'insérer l'analogie des peintres. Empédocle pose dans les vers 232308 du premier livre du Peri physeos les six principes du monde physique, c'est-à-dire les quatre éléments (feu, eau, terre et air) et les deux puissances cosmiques, l'Amour et la Haine, qui alternent leur domination dans un cycle qui va de l'Un au Multiple et du Multiple à l'Un. La fonction de l'Amour est de créer des liens entre des éléments de nature différente, celle de la Haine est de les dissocier. Chacune des phases du cycle donne lieu à une zoogonie, l'une sous l'emprise de l'Amour et l'autre sous celle de la Haine, comme le laissent entendre ces vers qui ont fait l'objet de longs débats (234-6 = B17.3-5):

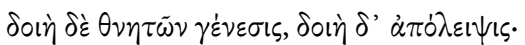

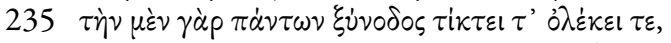
$\dot{\eta} \delta \dot{\varepsilon} \pi \dot{\alpha} \lambda \iota \nu \delta\left(\alpha \phi \nu 0 \mu \dot{\varepsilon} \nu \omega \nu \theta \rho \varepsilon \phi \theta \varepsilon i \tilde{\sigma} \alpha \alpha \delta \dot{\varepsilon} \pi \tau \eta^{17}\right.$.

16. Sur la position de l'ensemble Strasb. b, intercalé entre B21 et B23, voir supra n. 13.

17. C'est sans doute l'un des vers les plus problématiques du poème, tant par l'établissement du texte que par les interprétations divergentes qu'il a suscitées: $\dot{\eta} \delta \dot{\varepsilon} \pi \dot{\alpha} \lambda \iota \nu$ $\delta 1 \alpha \phi \nu 0 \mu \dot{\varepsilon} \nu \omega \nu+\theta p \nu \phi \theta \varepsilon \tilde{\sigma} \sigma \alpha \delta \rho \varepsilon \pi \tau \dot{\eta}$ †. La finale du vers est effectivement corrompue $(c f$.

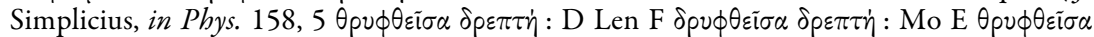
$\delta \rho \varepsilon \pi \tau \dot{\eta}$ Ald). Le vers, tel que transmis, n'est pas cohérent. Scaliger (le Jeune) a corrigé, par souci de métrique, $\delta \rho \varepsilon \pi \tau \dot{\eta}$ en $\delta เ \varepsilon \dot{\varepsilon} \pi \tau \eta$. Or cela n'offre que des termes convenant à une destruction, en aucune manière à une naissance, alors que le vers 235 évoque une double naissance et une double destruction. On s'attend à ce que les vers 236-237 explicitent les deux naissances et les deux destructions. L'énoncé du vers 236 évoque une première naissance et une première disparition, mais à la place d'un parallèle au vers 237 , on n'a que

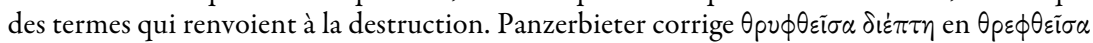
$\delta เ \dot{\varepsilon} \pi \tau \eta$. C'est sur ce vers que Panzerbieter - suivi par Zeller, Diels, Bignone, O'Brien, Wright, Inwood, Primavesi et al. - a appuyé la thèse de la «double zoogonie ». D'autres conjectures possibles : $\theta p \cup \phi \theta \varepsilon i \tilde{\sigma \alpha} \dot{\alpha} \pi \dot{\varepsilon} \sigma \tau \eta$ Sturz: $\theta \rho \cup \phi \theta \varepsilon \tilde{\imath} \sigma \alpha \delta_{1} \dot{\varepsilon} \pi \tau \eta$ Karsten, Diels Poet. 
Double est la naissance des choses mortelles, double leur disparition : L'une, la réunion de tous l'enfante et la détruit aussi bien,

L'autre, quand inversement ils se séparent, s'envole une fois qu'elles ont pris consistance.

Dans la phase de l'Amour croissant, qui va du Multiple à l'Un, les naissances s'expliquent par la réunion d'éléments différents. En vertu du cycle cosmique, la création du vivant n'est pas le but ultime de l'Amour. Il ne représente, au contraire, qu'une des phases du devenir dans le cadre d'une histoire qui s'achève par l'unification intégrale des quatre éléments dans la masse homogène de l'Un. Inversement, selon l'interprétation traditionnelle, la Haine n'est pas seulement destructrice, en ce sens que le morcellement de l'Un dont elle est responsable dans la phase suivante donne lieu à une zoogonie par séparation d'éléments; une séparation qui aboutit éventuellement, avec la progression de la Haine, à la disparition des diverses formes de vie. Cette interprétation suppose que la Haine crée des formes de vie au même titre que l'Amour, bien que nous n'ayons parmi les fragments qui nous sont parvenus aucun passage décrivant explicitement la formation des espèces vivantes par la Haine ${ }^{18}$. Bien au contraire, la physiologie du vivant est systématiquement présentée dans le poème physique comme l'œuvre d'Amour. Si notre monde se trouve, comme on a des raisons de le penser, dans la phase de la Haine croissante $^{19}$, l'interprétation traditionnelle se heurte à l'anomalie qui suppose qu'une doctrine rende compte exclusivement de formes de vie conçues dans une autre ère cosmique que la sienne. D'autre part, Empédocle exhorte son disciple à voir autour de lui les œuvres de l'Amour qui vivent encore dans ce monde ${ }^{20}$. Une solution élégante pour éviter de donner à la Haine une fonction créa-

Philos. : $\delta \rho \cup \phi \theta \varepsilon i \sigma^{\prime} a ̉ \pi \circ \delta \rho \dot{\pi} \pi \tau \varepsilon$ Bollack. Voir la discussion dans Martin et Primavesi 1999, p. 76 sq.

18. Empédocle mentionne de façon allusive des processus cosmologiques sous la Haine, sans cependant décrire des modes spécifiques de génération dans cette phase cosmique. Le témoignage d'Aétius (V 19.908d-e = A72) qui distingue quatre étapes de ce qui semble être un seul et même processus zoogonique a été souvent interprété comme regroupant deux zoogonies distinctes. Les quatre étapes mentionnées sont les suivantes: 1. membres séparés

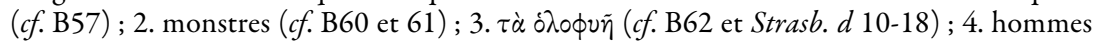
et femmes. Pour la plupart des spécialistes, les étapes 1 et 2 correspondent à la zoogonie sous l'Amour, tandis que les étapes 3 et 4 dépendent de l'action de la Haine. Ce schéma, qui distribue les générations sur deux courbes distinctes, pose le problème de la localisation de Sphairos, lequel n'est pas mentionné dans le témoignage d'Aétius, alors qu'il représenterait, dans l'hypothèse des deux zoogonies, une discontinuité majeure qui devrait figurer entre les deux zoogonies.

19. Cf. Aristote, $G C$ II, 6, 334a5-7 (= A42) : « [Empédocle] dit aussi que le monde est dans le même état maintenant, sous la Haine, que précédemment sous Amour. »

20. Cf. $291-295$ (= a (ii) 21-25); B21. 1 ; B71. 
trice symétrique de celle de l'amour est d'admettre avec Primaves ${ }^{21}$ que même sous la période de la Haine croissante c'est l'Amour qui, certes avec des forces diminuantes, demeure la puissance créatrice du vivant.

Empédocle distingue l'agir des deux puissances cosmiques de façon quasi systématique au moyen de termes composés : les mots construits avec $\sigma \dot{v} v$ - (乡ंv) désignent les processus placés sous l'emprise de l'Amour ${ }^{22}$, alors que des termes composés avec le préverbe $\delta \dot{\alpha}^{\alpha}$ - ont tendance à qualifier des processus cosmologiques placés sous l'action de la Haine $e^{23}$. Les deux forces sont décrites comme des entités mesurables, spatialement localisables et équipollentes. Bien que toutes deux agissent directement sur les éléments, une caractéristique importante les distingue : l'Amour agit de l'intérieur, il se tient « en eux 》 ( $\dot{\varepsilon} \nu \tau o i ̂ \sigma l v, 251=B 17.20)$, par opposition à la Haine qui est située à l'écart, « séparée d'eux » $(\delta i \chi \alpha \tau \tilde{\omega} \nu, 250=B 17.19)$. Cette inhérence qui lie l'Amour aux éléments lui donne un statut physique et poétique particulier. Outre le fait d'avoir l'Amour en nous, « implanté dans les

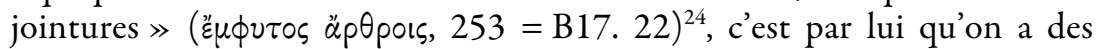
«pensées aimantes » et qu'on réalise des « œuvres de jonction » ( $\tau \tilde{\eta} \iota \tau \varepsilon$

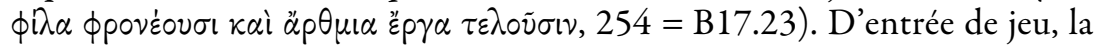
doctrine assigne à l'Amour un rôle d'artisan (ou d'artisane, en particulier sous les noms d'Aphrodite ou Cypris), mis en relief dans ces vers par les termes à racine * $\ddot{\alpha} \rho-:$ les jointures $(\ddot{\alpha} \rho \theta p \alpha)$ et les travaux des hommes ( $\ddot{\alpha} \rho-$ $\mu \ltimes \alpha$ है $p \gamma \alpha)$ - tout comme le char $(\ddot{\alpha} p \mu \alpha)$, métaphore du poème dans un autre passage (B3. 5) - témoignent d'une puissance d'ajustement ${ }^{25}$. Le modèle technique n'est cependant pas le seul à décrire dans le poème le mouvement des éléments sous l'emprise de l'Amour. Une autre métaphore, qu'Empédocle répète trois fois à proximité de l'analogie des peintres, affirme que tout provient des éléments «courant les uns à travers les

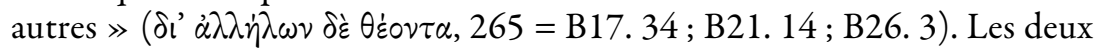
métaphores, la technique et la course, ne sont pas antinomiques, elles expriment toutes deux la tendance naturelle des éléments à se rejoindre. Ce qui les distingue, et qui justifie leur coexistence dans le même passage, est l'expression de points de vue différents par rapport au cycle cosmique. La métaphore de la «course» introduit un effet d'éloignement: sous

21. Mansfeld et Primavesi 2011, p. 403.

22. Cf. छ̇v en adverbe : $\mathrm{B} 21.8 ; \mathrm{B} 95 ; 252=\mathrm{B} 17.21 ;$ en composé $: 235=\mathrm{B} 17.4 ; 238$ $=\mathrm{B} 17.7 ; 294=\mathrm{a}$ (ii) $24 ; 300=\mathrm{a}$ (ii) $30 ; \mathrm{B} 20.2 ; \mathrm{B} 22.8 ; \mathrm{B} 26.5,7$; B35. 5, 6; B36; B53 ; B59. $2 ; \mathrm{B} 71.4 ; \mathrm{B} 98.1 ; \mathrm{B} 104$.

23. Cf. $233 ; 236 ; 241 ; 243 ; 248(=\mathrm{B} \cdot 17.2,10,12,17) ; 294=\mathrm{a}$ (ii) $24 ; 300=\mathrm{a}$ (ii) $30 ; \mathrm{B} 20.4 ; \mathrm{B} 21.7 ; \mathrm{B} 22.6 ; \mathrm{B} 26.9 ; \mathrm{B} 63$.

24. Dans la poésie archaïque, les jointures représentent l'endroit du corps par où le sentiment amoureux pénètre. Cf. Calame 1996, p. 28.

25. Voir aussi B35. 17 ; B71. 4 ; B107. 1. 
l'Amour, tout provient de la rencontre d'éléments, aussi bien les espèces vivantes que Sphairos; l'accent est mis sur l'attraction élémentaire pure et simple plutôt que sur le mode d'articulation ou la proportion des mélanges. De son côté, la métaphore artisanale introduit une forme de finalisme esthétique là où il n'existe $a$ priori qu'une force d'attraction entre éléments différents; ce point de vue permet d'explorer de près la complexité du vivant par analogie avec des processus techniques. Le choix d'Empédocle de manier simultanément les métaphores de la course et de la technique peut être interprété comme un rappel que l'Amour est au service de l'homogénéité de l'être par le biais du mélange des éléments «courant les uns à travers les autres ». L'unité relative de chacun des chefs-d'œuvre techniques de l'Amour n'est en fait jamais que l'anticipation de sa disparition à venir dans l'unité de Sphairos, où provisoirement s'achève la « course ».

Comparans et comparandum : la difficulté des duels

Destinée à éclairer le processus de génération des espèces vivantes à partir des divers mélanges élémentaires, l'analogie des peintres se prête aisément comme illustration des compétences démiurgiques de l'Amour. Mais si Empédocle avait voulu éclairer cette seule puissance démiurgique, pourquoi a-t-il construit l'analogie avec plusieurs peintres (le sujet et les verbes sont au pluriel) au lieu d'un seul ? Pourquoi les procédés techniques des peintres sont-ils exprimés par des participes au duel $(\delta \varepsilon \delta \alpha \tilde{\omega} \tau \varepsilon ; \mu \varepsilon i \xi \alpha \nu \tau \varepsilon$;

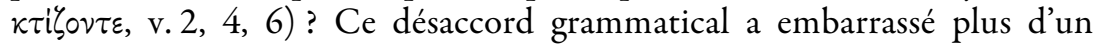
commentateur. Pour Jean Bollack, « il n'y a aucune idée de dualité » dans ces duels-là ; ces désinences ont une fonction stylistique qui consiste à mettre en relief les moments essentiels dans la fabrication des vivants ${ }^{26}$. M. R. Wright, de son côté, trouve les duels assez énigmatiques et propose de les interpréter comme des pluriels sur la base d'exemples homériques ${ }^{27}$. S'il est vrai que les duels dans la poésie archaïque peuvent dans certains cas être interprétés comme de simples pluriel $\mathrm{s}^{28}$, cette lecture est insatisfaisante dans la mesure où elle ne rend pas compte de l'intentionnalité sémantique qui s'exprime dans la récurrence de cette désinence grammaticale dans le fragment B23. En effet, les duels sont suffisamment rares dans les fragments d'Empédocle pour qu'on les considère comme des objets herméneutiques à part entière. Â part les trois cas en question, qui se trouvent répartis sur cinq vers successifs, il n'existe qu' un autre exemple de duel, dans un passage assigné aux Catharmes (B137. 6). En outre, dans le cas de $\delta \varepsilon \delta \alpha \tilde{\omega} \tau \varepsilon$ (B23. 2),

26. Bollack 1969, vol. 3, p. 122 .

27. Cf. Il. III. 279 ; VIII. 186 ; XVI. 371 ; XVII. 387. Wright 1981 ajoute : « The duals here may have resulted from Simplicius MSS reading of $\ddot{\alpha} \mu \omega \omega$ for $\dot{\alpha} \mu \phi i$, or perhaps Empedocles is allowing himself a striking flexibility in the forms 》 (p. 179-80).

28. Voir la discussion dans Chantraine 1948, vol. 2, p. 26-28. 
le duel ne serait même pas requis par la métrique. Plus récemment, Simon Trépanier et David Sedley ${ }^{29}$, suivant chacun des approches différentes, ont remis en question l'interprétation faible des duels dans B23. Leurs arguments plaident pour l'attribution d'une signification théorique à ces formes grammaticales, en l'occurrence les duels de B23 renverraient aux deux puissances cosmiques, l'Amour et la Haine. La piste est intéressante dans la mesure où elle sort les duels de l'anonymat, mais elle attribue du coup un rôle démiurgique à la Haine qui est pour le moins problématique.

Le premier argument en faveur d'une lecture de l'analogie des peintres élargie aux deux puissances cosmiques fut présenté par Trépanier. Son hypothèse générale est que la Haine joue un rôle actif dans la zoogonie de l'Amour. Pour le démontrer, il s'appuie sur les reprises formulaires que nous avons déjà mentionnées (269-272 B21. 9-12 B23. 5-8), dont la compréhension est conditionnée par le référent que l'on attribue au

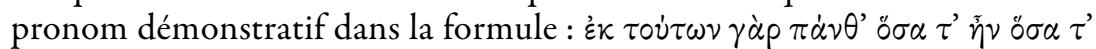

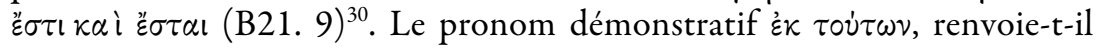
seulement aux quatre éléments ou plutôt à l'ensemble des six principes (l'Amour et la Haine en plus des éléments) ? Selon Trépanier, le fait que le pronom apparaisse dans un contexte où il est question des six principes du devenir indique qu'il se réfère effectivement à l'ensemble de ces principes. C'est de leur action conjointe que proviendrait le vivant. Cette lecture se trouve à ses yeux confirmée par les participes au duel dans l'analogie des peintres : ce sont l'Amour et la Haine, agissant simultanément sur les quatre éléments, qui produisent la diversité des espèces vivantes. Trépanier attribue à la Haine un rôle créatif dans la zoogonie : en limitant les portions d'éléments dans les mélanges ( $2 \lambda \lambda \alpha \delta^{\prime} \dot{\varepsilon} \lambda \dot{\alpha} \sigma \sigma \omega, \mathrm{B} 23.4$ ), elle contribue à leur harmonie ${ }^{31}$.

L'interprétation de Trépanier soulève un certain nombre de problèmes. En premier lieu, il n'est pas certain que le référent du pronom démonstratif

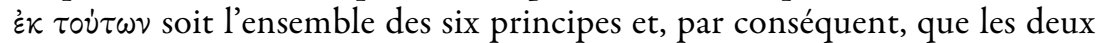
puissances agissent ensemble sur les éléments. La formule zoogonique $\dot{\varepsilon} \kappa$

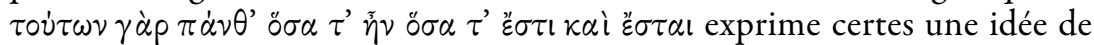
totalité, comme en témoigne l'inventaire du monde qui en découle dans les vers suivants (B21. 10-12). Cependant, si l'on se tient à la correspondance entre le comparandum et le comparans, c'est-à-dire entre '́ck $\tau 0 \dot{\tau} \tau \omega \nu \pi \dot{\alpha} \nu \theta^{\prime}$

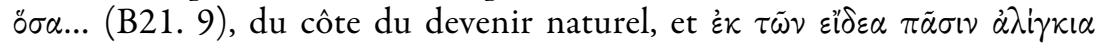

29. Trépanier 2003 ; Sedley 2007, p. 57-59.

30. La question concerne aussi la variante sous la forme de pronom relatif $\dot{\xi} \xi \tilde{\omega} \nu$ dans Strasb. $a,(269=a$ (i) 8), qu'Aristote mobilise comme témoignage d'une zoogonie sous la Haine dans Metaph. B4, 1000a26 sqq.

31. Trépanier 2003, p. 33-36. Cette interprétation a été reprise par Ierodiakonou 2005; Warren 2007, p. 141 ; Wersinger 2008, p. 89 ; Skarsouli 2009. 


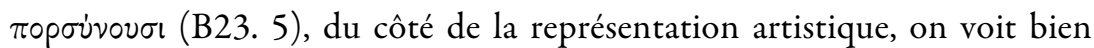
que $\dot{\varepsilon} \kappa \tau \circ \dot{v} \tau \omega \nu \pi \dot{\alpha} \nu \theta^{\prime}$ ö $\sigma \alpha[\ldots]$ ne peut pas recouvrir la totalité des six principes, mais seulement les quatre éléments. Dans le comparans, en effet, les peintres ont un statut différencié de celui des pigments, ils apparaissent comme une cause efficiente, séparée du matériau sur lequel ils agissent. Si le comparans a un effet heuristique sur le comparandum, comme on a des

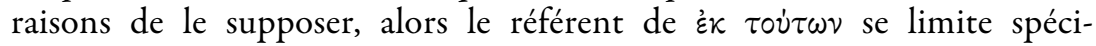
fiquement aux quatre éléments. L'un des aspects de la doctrine que l'analogie des peintres permet de comprendre est que les espèces vivantes proviennent effectivement des quatre éléments, mais pas nécessairement de l'action conjointe des deux puissances, comme le suppose la lecture de Trépanier. L'autre objection soulevée par cette interprétation concerne le rôle de la Haine dans la conception du « mélange harmonieux ». L'Amour a-til besoin de la coopération de la Haine pour réussir un mélange complexe d'éléments ? Pour autant que l'on puisse en juger par les fragments qui nous sont parvenus, la notion d'harmonie est exclusivement employée dans le poème comme un attribut de l'Amour ${ }^{32}$. D'autre part, les fragments B96 et B98, qui décrivent respectivement la composition des os et des chairs, ne prêtent aucun rôle, fût-il implicite, à la Haine dans la conception des mélanges. Le juste dosage des éléments fait partie de la metis zoogonique d'Aphrodite; à aucun moment le savoir-faire technique de la déesse n'implique quelque chose comme une contribution sous-entendue de la Haine.

De son côté, Sedley s'est aussi interrogé sur le sens des trois participes au duel. Comme l'interprétation de Trépanier, sa lecture trahit ce que l'on pourrait appeler un «horizon d'attente » qui d'une certaine manière la prédispose à trouver dans l'analogie la confirmation d'une interprétation déjà élaborée de l'ensemble de la doctrine. Sedley défend une interprétation originale de la double zoogonie selon laquelle les deux processus auraient lieu dans le même monde. Il n'y a pas de discontinuité entre les phases zoogoniques de l'Amour et de la Haine, seulement une alternance à l'intérieur d'un cycle mineur, qui néanmoins est assujetti au mouvement d'un cycle cosmique majeur qui va de l'Un au Multiple et du Multiple à l' $\mathrm{Un}^{33}$. Le monde présent contiendrait des créatures provenant des deux zoogonies. Les multiples exhortations d'Empédocle à Pausanias à voir autour de lui les témoignages vivants de la doctrine physique indiquent ${ }^{34}$, selon Sedley, que nous sommes en présence de deux types d'espèces, les unes nées sous la Haine, les autres sous l'Amour. Pour classer ces espèces en fonction de la

32. Cf. ad B18; B27.3.

33. Sedley 2007, p. 33-52.

34. Cf. $291-295$ (= a (ii) 21-25) ; B21. 1. 
zoogonie d'où elles proviennent, Sedley s'appuie sur le témoignage d'Aétius (V 19. 908 d-e = A72), qu'il met en rapport avec l'énumération des créatures dans l'inventaire du monde (B21. 9-12 B23. 5-8 $)^{35}$. Ainsi, les arbres, les hommes et les femmes, nommés en premier, sont le produit de la Haine, tandis que les bêtes, les oiseaux, les poisons et les dieux, nommés dans les deux vers suivants, proviennent de la zoogonie de l'Amour. Ce schéma trouve aux yeux de l'auteur une illustration dans l'analogie des peintres. Les duels signifieraient le travail créatif des deux puissances, mais à la différence de Trépanier, pour qui il y a simultanéité dans leur action, pour Sedley, la démiurgie des deux puissances est consécutive. L'ordre d'apparition des créatures qui se dégage de l'inventaire du monde indique que la Haine « peint » d'abord (arbres, hommes et femmes, v. 6) et l'Amour ensuite (bêtes, oiseaux, poisons et dieux, v. 7-8). Ce n'est pas ici le lieu de discuter l'interprétation de l'ensemble du cycle proposée par Sedley. On dira seulement que l'analogie des peintres semble mal adaptée à illustrer cette vision du cycle cosmique, notamment la partie de la comparaison qui correspondrait à la zoogonie de la Haine. Comme le reconnaît l'auteur luimême, « on the one hand, the two artists mix their colours, and, correspondingly, Love's and Strife's products are mixtures. On the other, Strife's contribution consists in progressive separation, and its comparison to an artist, if made entirely explicit, would therefore give undue emphasis to the loosest part of the analogy $»^{36} . \mathrm{Si}$, comme je le pense, les comparaisons d'Empédocle - à l'instar de celles d'Homère, dont elles héritent en partie la structure et le mode de fonctionnement ${ }^{37}$ - étaient des artefacts poétiques destinés à une audience avertie, capable d'élucider le jeu complexe de correspondances entre comparans et comparandum, une analogie aussi importante que celle des peintres pouvait difficilement admettre un contresens comme celui que signale Sedley.

Mon hypothèse est que l'analogie des peintres décrit un processus dont seule la puissance cosmique d'Amour est responsable ; la comparaison recèle suffisamment d'indices en ce sens. Le premier concerne l'objet même sur lequel travaillent les peintres: les $\dot{\alpha} \nu \alpha \dot{\eta} \mu \alpha \tau \alpha$. Le mot désigne les offrandes votives en général et dans ce contexte particulier les peintures que l'on déposait dans les temples à l'intention d'une divinité ${ }^{38}$. Il se trouve que ces représentations picturales ont une fonction symbolique capitale dans la pensée religieuse d'Empédocle, comme en témoignent les peintures évo-

35. Sur le témoignage d'Aétius, voir supra, n. 18.

36. Sedley 2007, p. 59, n. 88.

37. Les travaux de Kranz 1938, de Snell 1953, p. 213-223 et de Bollack 1965, p. 295 310 , restent des références incontournables pour comparer le mode de fonctionnement des analogies homériques avec celles d'Empédocle.

38. Voir Rouse 1902, p. 323-324. 
quées dans un passage des Catharmes (B128) où l'on décrit le rituel qui se substitue au sacrifice sanglant dans l'âge de Cypris. Dans un âge où règne la paix, en effet, les hommes cherchent les faveurs de la divinité non par le versement de sang, mais «par des images pieuses $(\dot{\alpha} \gamma \dot{\alpha} \lambda \mu \alpha \sigma v)$, / par des

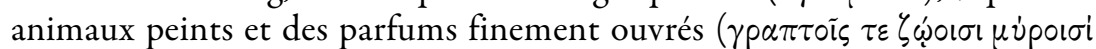
$\tau \varepsilon \delta \alpha ı \delta \alpha \lambda \varepsilon \delta \delta \delta \mu \circ \varsigma) \gg(B 128.4-5)$. L'art pictural apparaît ici lié à des pratiques religieuses qui sont indissociables d'une forme de domination de l'Amour. Conséquemment, le choix même du thème des peintres comme analogon de la génération du monde en B23 pourrait avoir, en plus d'une utilité heuristique, une signification axiologique dans le cadre de la doctrine physique $^{39}$. Si, comme nous l'avons déjà suggéré, la technique chez Empédocle est à la fois une manifestation de la puissance de l'Amour et son analogon privilégié, alors les «peintres » qui, sur un plan cosmique, « composent des figures semblables à toutes les choses » (B23. 5) deviennent en quelque sorte le modèle de ces autres peintres réels qui, à l'âge de Cypris, représentent des animaux affranchis du sacrifice sanglant. De son côté, le choix du verbe $\pi$ okki $\lambda \lambda \omega$ pour désigner l'action des peintres n'est pas dépourvu de connotations à la fois démiurgiques et cosmologiques. Le verbe n'apparaît qu'une seule fois dans l'épopée, pour décrire la fabrication par Héphaïstos de l'une des scènes du bouclier d'Achille (Il. XVIII, 590), lequel déploie, comme on sait, une représentation totalisante du monde. L'autre occurrence connue antérieure à Empédocle se trouve chez Phérécyde (DK 7 B2), où le verbe désigne l'action du dieu Zas brodant sur une robe la Terre et l'Océan. D'entrée de jeu, le cadre narratif de l'analogie met en scène des peintres dans une situation qui s'avère incompatible avec un supposé rôle démiurgique de la Haine.

Qu'en est-il des duels qui expriment les procédés techniques des peintres ? Les trois participes marquent, comme l'a signalé Bollack à juste titre, des moments fondamentaux de la création picturale ${ }^{40}$. Le premier, $\delta \varepsilon$ $\delta \alpha \tilde{\omega} \tau \varepsilon$, « instruits 》 (B23. 2), désigne le savoir-faire des artisans. Dans l'épopée, le verbe $\delta \dot{\alpha} \omega$ s'applique à l'aède $(O d$. XVII, 519) et à l'orfèvre $(O d$. VI, 233); dans les deux cas, un agent divin instruit l'artisan. Chez Empédocle, l'agent qui instruit les peintres est leur propre habileté (íđò $\left.\mu \eta \dot{\tau} \tau)_{5}\right)$. Intelligence technique par excellence ${ }^{41}$, la metis constitue aussi l'es-

39. Sur le rapport entre la « nécessité » qui gouverne le cycle cosmique et celle qui règle le cycle eschatologique des Catharmes, voir Laks 2013. Dans mon interprétation, l'analogie de la peinture dans le poème physique fait signe vers une forme symbolique qui dans le poème éthique permet aux pratiques religieuses de se soustraire au sacrifice et donc, dans une certaine mesure, d'infléchir la « nécessité » qui règle le récit eschatologique.

40. Cf. supra, n. 26.

41. Dans l'épopée, l'opposition entre l'habileté ( $\mu \eta \dot{\tau} \iota)$ et la force ( $\beta i \eta)$ structure la querelle entre Ulysse et Achille. L'habileté apparaît souvent comme un moyen qui s'oppose à la 
sentiel de la promesse de connaissance faite au disciple dans le proème:

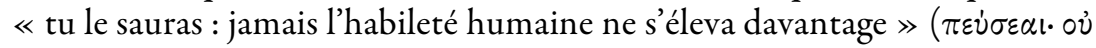

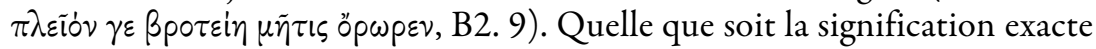
de la metis dans la doctrine d'Empédocle, il est clair que l'habileté des peintres dont il est question dans le micro-récit qui encadre l'analogie renvoie indirectement au thème du proème : puisqu'ils ont déjà acquis la metis dans leur art, les peintres sont déjà en quelque sorte initiés dans la doctrine d'Empédocle. Après la reconnaissance du savoir-faire, vient le passage à l'œuvre, marqué en l'occurrence par le deuxième participe au duel : ápuovin $\mu \varepsilon i \xi \alpha \nu \tau \varepsilon$, « mélangeant harmonieusement [les pigments] ${ }^{42} \gg(B 23.4) . \mathrm{Le}$ mélange est, comme nous l'avons déjà souligné, le mode d'action privilégié de l'Amour ${ }^{43}$. En ce sens, l'un des points fondamentaux de la doctrine physique que l'analogie des peintres était sans doute en mesure d'éclairer pour les auditeurs est celui de la nature du mélange élémentaire. À quoi se réfère Empédocle quand il parle de «mélange harmonieux» comportant «tantôt plus tantôt moins de parts »? L'enjeu des vers B23. 3-5 est de mettre en évidence par le biais de la technique picturale que le mélange est un agrégat d'éléments à l'intérieur duquel les composants conservent leurs qualités ${ }^{44}$. Le principe qui veut que les éléments conservent leur identité quel que soit le mélange auquel ils participent est par ailleurs clairement énoncé dans les deux vers qui précèdent l'analogie : $\alpha \dot{\tau} \tau \grave{\alpha} \gamma \alpha \grave{\alpha} \rho \tilde{\varepsilon} \sigma \tau \imath \tau \tau \alpha \tilde{\nu} \tau \alpha, \delta$ '

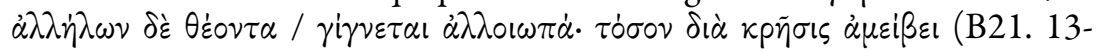
14). Afin d'illustrer ce principe, Empédocle transpose les normes opératoires de l'art pictural à la génération des formes organiques. L'avantage heuristique de cet analogon est double. D'une part, la peinture du $V^{e}$ siècle av. J.-C., offrait un exemple éminent de la façon dont la combinaison d'un

force dans des activités dirigées à une fin déterminée. Les exemples choisis par Homère pour illustrer l'avantage de l'habileté sur la force (Il. XXIII, 313-318), à savoir l'abattage d'un arbre, le pilotage d'un navire et la conduite d'un char, laissent entendre qu'elle ne relève pas exclusivement d'un refoulement de la rationalité objectivante, comme l'ont prétendu Detienne et Vernant 1974. Pour ce qui est de l'étymologie, Chantraine montre que le terme $\mu \dot{\eta} \tau \iota \zeta$ « qui s'applique à l'intelligence pratique, parfois à la ruse, est issu d'une racine verbale qui signifie "mesurer" : mesurer implique calcul, connaissance exacte ». Cette ambiguïté entre connaissance exacte et ruse est patente chez Pindare, où la $\mu \dot{\eta} \tau \iota \varsigma$ peut désigner tour à tour la ruse du renard au combat (Isth. IV, 46-47), l'intelligence du stratège (Ol. XIII, 50), la prudence politique (Pyth. IV, 262) et le talent de composition que le poète sollicite de la Muse (Ném. III, 9). La $\mu$ ì $\tau$ chez Empédocle a été relativement peu étudiée. Kingsley 2003 aborde la question dans le sillage de Detienne et Vernant.

42. Le terme $\phi \dot{\alpha} p \mu \alpha \kappa \alpha$ désigne dans ce contexte les pigments utilisés dans la peinture. $C f$. Hérodote, I, 98 ; Platon, Resp. 420c, Crat. 424e, Pol. 277c.

43. Cf. $\mu i \xi ı$ : B8. $3 ; \mu i \sigma \gamma \omega:$ B35. 7 et $16 ; \mu \varepsilon i \gamma \nu \nu \mu$ : B9. 1.

44. Voir l'interprétation de ces vers par Wright 1981, p. 38-39, 180 et Mourelatos 1987, p. 141 n. 5. 
nombre limité d'éléments - quatre, en principe ${ }^{45}$ - donne lieu à la prolifération des formes et des couleurs. De l'autre, la peinture de cette époque opérait selon une technique de stratification de couches de couleur qui rendait le processus de mélange en tout point discernable. L'application des couleurs s'effectuait aplat par aplat, la variation chromatique s'obtenait par la superposition des touches de couleurs différentes, en ajoutant par assombrissement ou éclaircissement de la couleur, les ombres et les lumières ${ }^{46}$. La pertinence de l'analogie des peintres pour illustrer la nature du mélange empédocléen est confirmée indirectement par Aristote lorsque, sous la forme d'une autre analogie technique, il décrit le mélange des éléments dans cette doctrine comme la construction d'un mur à partir de briques et de pierres $(G C, 334 \mathrm{a} 27-31=\mathrm{A} 43)$. Le qualificatif $\mathrm{d}$ ' « harmonieux » qu'Empédocle donne au mélange des couleurs trahit par ailleurs une conception de la peinture comme technique d'assemblage, d'accord heureux, qui relève, comme nous l'avons déjà dit, de la domination d'Aphrodite. Enfin, le troisième participe au duel, $\kappa \tau i \zeta o v \tau \varepsilon$, «créant » (B23. 6), marque l'étape proprement démiurgique de la représentation picturale. Le verbe $\kappa \tau i \zeta \omega$ est le pendant « technique » de $\beta \lambda \alpha \sigma \tau \dot{\alpha} \nu \omega$ (B21. 10) qui, du côté du comparandum, désigne le devenir naturel. Avec la réalisation des formes ( $\varepsilon$ 'i $\delta \varepsilon \alpha)$, s'achève la comparaison, dont la fonction principale est de mettre en évidence la source du devenir ( $\dot{\varepsilon} \kappa \tau \dot{\tau} \tau \omega \nu$, B21. $9 \sim \pi \eta \gamma \eta \dot{\eta}$, B23. 10) et le mode opératoire de l'Amour.

L'analogie des peintres n'implique à aucun moment l'intervention de la Haine, on peut par conséquent exclure l'hypothèse que les duels renvoient aux deux puissances cosmiques travaillant soit simultanément (Trépanier), soit successivement (Sedley). En revanche si l'Amour est, comme je le pense, la puissance cosmique dont l'analogie éclaire le mode d'action, alors les duels devraient signifier un aspect lié à cette action. En ce sens, ce qui caractérise d'un point de vue instrumental l'agir d'un artisan dans l'épopée est précisément qu'il travaille avec les mains, dont la caractéristique saillante est d'être double. Ce n'est donc sans doute pas un hasard si les mains, ou plus exactement les paumes, de la déesse sont mentionnées à deux reprises dans des contextes zoogoniques ${ }^{47}$. Le premier passage, B75, est cité par

45. Voir supra n. 12.

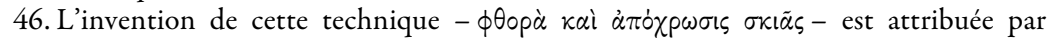
Plutarque à Apollodore, Glor. Ath. 346A. Le terme $\phi \theta 0 p \dot{\alpha}$, « corruption », désigne techni-

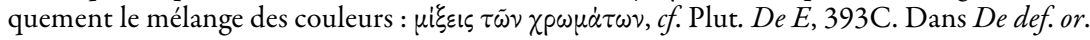
436B-C, on parle de couleurs « broyées et mélangées ensemble » comme un préalable à la

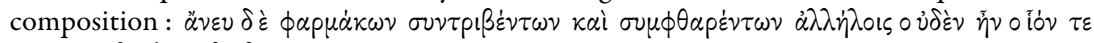

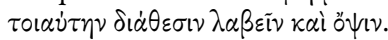

47. Je dois cette remarque fondamentale pour mon interprétation à Jean-Claude Picot. 
Simplicius (in de Caelo, 530, 9 sq. ${ }^{48}$, sans glose, à propos du nom de Cypris. Il décrit la confection d'un organe du corps ; les mains de la déesse forment à partir du mélange d'éléments des tissus plus ou moins denses :

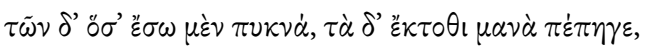

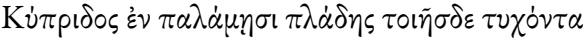

Chez ceux-ci, tout l'intérieur acquit dureté en se caillant, et l'extérieur souplesse,

Ayant reçu dans les paumes de Cypris cette moiteur-là.

L'autre fragment où sont mentionnées les paumes de la déesse est B95, cité par Simplicius à propos de l'œil et de la vision (in de Caelo, 529. 26) :

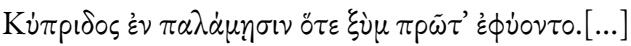

Quand dans les paumes de Cypris ils croissaient pour la première fois.

Dans ces deux passages, la mention des paumes de la déesse se fait par un

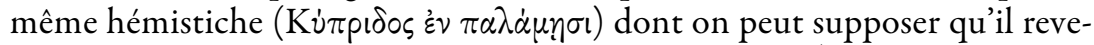
nait comme un leitmotiv dans la zoogonie de l'Amour ${ }^{49}$. Le modèle technique déployé dans cette partie de la doctrine focalise l'attention de l'auditoire vers ce qui arrive dans les paumes de la déesse, tout comme, dans l'analogie des peintres, les duels mettent en relief des procédés qui impliquent directement les mains des artisans: le savoir-faire, le mélange des couleurs et la création proprement dite. Signalons aussi qu'outre les mains de la déesse, le mot $\pi \alpha \lambda \alpha \dot{\alpha} \alpha$ ı désigne métaphoriquement chez Empédocle l'appareil sensoriel des humains (B2. 1 et B3.9) : nous saisissons le monde à travers des paumes répandues dans nos membres. C'est dire à quel point la représentation des mains de l'artisan joue un rôle structurant dans la doctrine physique.

La mise en valeur des paumes est par ailleurs courante dans l'épopée, où les mains constituent non seulement l'attribut par excellence de l'artisan, mais aussi la manifestation même de son savoir-faire ${ }^{50}$. Un passage célèbre de l'Iliade (III, 125-28) illustre mieux que tout autre le rôle symbolique des mains ; il s'agit de la scène où Hélène est en train de broder sur un manteau

48. Le deuxième vers du passage est cité ailleurs (in Phys. 331, 9) pour souligner, à l'appui d'Aristote, le rôle dévolu au hasard.

49. Voir Bollack 1969, vol. 3, p. 391.

50. Voir les quelques comparaisons techniques d'Homère analysées par Fränkel 1921, p. 52-59, où la main de l'artisan n'est jamais absente, en particulier Il. XV, 411-13. Les paumes de l'artisan sont mentionnées chez Hésiode dans l'épisode de la fabrication de la couronne de Pandore par Héphaïstos, Theog. 578-580. Voir aussi Scutum, 219 et 320. Pindare utilise le mot $\pi \alpha \lambda \dot{\alpha} \mu \alpha \iota$ pour l'intervention bénéfique ou fatale de dieux, cf. Ol.X, $21 ;$ Pyth. I, 48. Chez Hérodote (VIII, 19, 1), les paumes deviennent une métaphore de l'habileté, du maniement. 
la représentation des malheurs de la guerre de Troie qui sont aussi l'objet du récit principal de l'Iliade:

Elle (scil. Iris) la trouva dans la demeure; elle tissait une grande pièce d'étoffe,

Un manteau pourpre, brodant les luttes nombreuses

Des cavaliers Troyens et des Achéens à la cuirasse de bronze

Que par sa faute ils enduraient sous les paumes d'Arès.

Dans ce passage, l'œuvre réalisée par les paumes d'Arès, la divinité guerrière, est simultanément reproduite par les mains d'Hélène dans sa broderie et par le poète de l'Iliade dans son récit. Il s'agit là, pour le coup, d'une démiurgie de Neikos : c'est la destruction même de la race des Héros sous les paumes d'Arès que les arts sont amenés à représenter. Chez Empédocle, les mains des peintres, tout comme les vers du poète, reproduisent ce que les paumes de Cypris réalisent sur un plan cosmique. Ce qui est frappant dans les deux cas est la mise en relation explicite entre les arts visuels, la poésie et l'action d'une divinité. L'artisan, qu'il soit poète, peintre ou brodeuse, refait les gestes manuels d'un dieu dont résulte un certain ordre du monde.

Pour revenir au fragment B23, peut-on inférer, à partir de ces exemples, que les duels dans l'analogie des peintres font allusion aux mains de la déesse artisane ? Comme on sait, les duels désignent en général des choses qui vont naturellement par deux, sans que ce soit systématique ( $\chi \varepsilon i \bar{p} \varepsilon, \pi \dot{\eta} \chi \varepsilon \varepsilon$, $\tau \dot{\varepsilon} v 0 \nu \tau \varepsilon, \ddot{\omega} \mu \omega, \mu \eta \rho \dot{\omega}, \grave{o} \sigma \sigma \varepsilon$, ỏ $\phi \theta \alpha \lambda \mu \dot{\omega}$, etc.). Chez Homère, comme chez Empédocle, le duel n'est jamais obligatoirement requis pour ce type de couple, qui peut être exprimé au pluriel ${ }^{51}$, comme en témoigne justement l'exemple des paumes. En même temps, le duel peut être un trait stylistique qui sert à créer ou à mettre en relief un couple ${ }^{52}$. C'est dans ce sens, me semble-t-il, qu'il convient d'interpréter les duels dans la comparaison des peintres. Puisque l'analogie ne peut concerner que la zoogonie de l'Amour, je ne vois pas de meilleur candidat comme référent de la désinence que les deux paumes de Cypris; les duels devaient rappeler à l'oreille avertie que les peintres ne font qu'imiter ce que les paumes de la déesse réalisent sur un plan cosmique. Le désaccord grammatical entre le sujet que sont les peintres $(\gamma \rho \alpha-$ $\phi \dot{\varepsilon} \varepsilon \zeta$, au pluriel) et les actions qu'ils mènent ( $\delta \varepsilon \delta \alpha \tilde{\omega} \tau \varepsilon, \mu \varepsilon \dot{\xi} \xi \alpha \nu \tau \varepsilon, \kappa \tau i \zeta o v \tau \varepsilon$, au

51. Cf. Monro 1891, p. 162, \$2.

52. Dans la poésie homérique : deux guerriers sur un char (Il. V, 244, 272, 568), les deux sources du Scamandre (Il. XXII, 147), deux lutteurs (Il. XXII, 707), deux danseurs (Od. VIII, 378), deux sirènes (Od. XII, 52), etc. 
duel) est au service d'un raisonnement analogique sophistiqué : alors que le pluriel désigne l'ensemble des peintres, les duels focalisent l'attention sur les deux mains d'un artisan particulier, instruites en l'occurrence par la metis

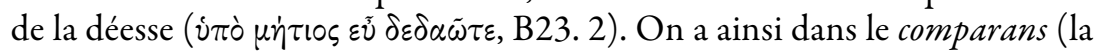
représentation picturale) un reflet anticipé du comparandum (la puissance cosmique), comme si la sphère de la technique était déjà en quelque sorte formée par celle de la nature.

\section{II}

Je voudrais pour conclure examiner le rapport qu'Empédocle construit dans le fragment B23 entre peinture, discours ( $\left.\mu \tilde{v} \theta_{\circ \varsigma}\right)$ et monde phénoménal, au regard des courants poétiques et philosophiques de son époque. Contrairement à d'autres images artisanales employées par Empédocle, qui relèvent d'une longue tradition poétologique, comme la poterie et la menuiserie $^{53}$, la représentation de la peinture apparaît relativement tard dans la poésie archaïque. De fait, pour un poète de la première moitié $\mathrm{du}^{\mathrm{e}}$ siècle av. J.-C., ériger l'art pictural en analogon de la génération des espèces vivantes, au même titre que le récit cosmologique qui en rend compte, n'est pas un geste dépourvu d'une certaine intention polémique. Au-delà de son utilité heuristique première dans le cadre de la doctrine physique, l'analogie des peintres construit un rapport spécifique entre les sphères cosmologique et symbolique (poétique et picturale) par lequel Empédocle se démarque à la fois de la tradition lyrique et de la spéculation philosophique en vers. L'emploi de l'analogie des peintres produit effectivement chez Empédocle un double écart réflexif par rapport aux visées propres de deux formes de discours qui lui sont contemporaines: d'une part, la poésie lyrique, notamment Simonide et Pindare, qui hiérarchise les formes symboliques de la parole et de l'image picturale en fonction des pratiques commémoratives; de l'autre, le poème philosophique de Parménide, qui soulève la question de la ressemblance entre l'objet même du discours (l'étant) et l'image qu'il construit de cet objet (la sphère). Mon hypothèse est que l'analogie des peintres touche simultanément deux problématiques distinctes mais contemporaines, travaillées respectivement par deux courants de la poésie archaïque : l'une concerne la relation entre formes symboliques et pratiques sociales instituées, l'autre, la fonction de l'image dans un discours qui se donne pour objet la totalité du réel.

La rivalité entre poésie et peinture: le rapport aux poètes lyriques

Pour mieux situer historiquement la nouveauté que représente l'analogie des peintres dans le domaine de la poésie archaïque, il convient de 
rappeler qu'avant d'être philosophique, la réflexion sur le rapport entre la parole et l'image picturale est poétique. Des comparaisons explicites entre ces deux formes symboliques sont déjà présentes dans la poésie lyrique dans la première moitié du $\mathrm{V}^{\mathrm{e}}$ siècle av. J.-C, au moment où Empédocle commence sa carrière. Élaborées notamment par Simonide et Pindare, ces comparaisons opposent sur un plan corporatiste la poésie et les arts visuels; plus exactement, il s'agissait chez les poètes lyriques de présenter la parole comme le meilleur moyen d'immortaliser un événement, au détriment de la matérialité de la peinture et de la statuaire. Le plus ancien témoignage que nous ayons de ce débat est un célèbre aphorisme attribué par Plutarque à Simonide, qui dit: «la peinture est une poésie muette et la poésie une

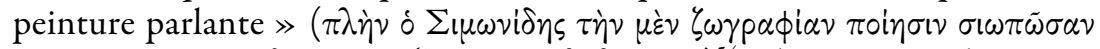
$\pi \rho \circ \sigma \alpha \gamma \circ p \varepsilon \dot{\varepsilon} \varepsilon l, \tau \eta \dot{\nu} \delta \delta \dot{\varepsilon} \pi \circ i \eta \sigma \omega \zeta \omega \gamma p a \phi i \alpha \nu \lambda \alpha \lambda \circ \tilde{\nu} \sigma \alpha \nu)^{54}$. Plutarque cite le propos de Simonide pour illustrer non pas une rivalité corporatiste, mais, dans une optique péripatéticienne, sa propre conception de la vivacité du style littéraire (enargeia) ${ }^{55}$. Dans ce sillage, l'aphorisme a parfois été interprété anachroniquement comme une anticipation de la théorie aristotélicienne de l'unité des arts imitatifs ${ }^{56}$. Or, quelle que fût sa formulation originelle ${ }^{57}$, il paraît plus probable que le propos de Simonide exprimait ironiquement une rivalité artisanale plutôt qu'une forme de spéculation esthétique. Comme l'a suggéré Andrew Ford, il convient d'interpréter le propos de Simonide à la lumière des odes de Pindare où le poète affirme la supériorité de la parole par rapport à la statuaire, à laquelle il reproche son immobilité et son silence $^{58}$. Si les œuvres d'art («poésie muette ») sont un moyen fréquent d'immortaliser une victoire, pour un chanteur d'épinicies, le poème («peinture parlante ») représente l'incarnation même de la renommée $(\kappa \lambda \dot{\varepsilon} \circ \varsigma)$. En ce sens, le propos de Simonide constitue non pas une première

54. Simonide, T101 Poltera (= 47b Campbell), Plut. Glor. Ath. 3 [346F]. Cf. Id. Aud. poet. 3 [18A], Adul. ab amico 15 [58B], Quaest. conv. 9, 15 [748A].

55. Plutarque mobilise effectivement l'aphorisme de Simonide pour un éloge du style « pictural » de Thucydide.

56. Thayer 1975, p. 14. Aristote, Poet. 48a1-9, fait de la peinture un art mimétique au même titre que la poésie et le théâtre. Pour appuyer la thèse de Simonide comme inventeur

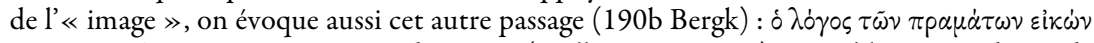
દ̇ $\sigma \tau \imath$, qui n'est attesté que très tardivement (Psellus, 821 Migne) et semble être un dérivé de l'aphorisme sur la poésie et la peinture.

57. On a des raisons de penser que la version de Plutarque est une reformulation des ipsissima verba de Simonide. Lanata 1963, p. 68, remarque que l'emploi de $\pi$ oin « poésie » n'est pas attesté avant Hérodote. Lucas 1968, p. 269 n. 2, signale de son côté que l'usage par Plutarque de $\lambda \alpha \lambda \varepsilon \tilde{\nu}$ comme synonyme de $\lambda \dot{\varepsilon} \gamma \varepsilon i \nu$ semble postérieur à Simonide. Sur la diction particulière des apophthegmata de Simonide, voir Wilamowitz-Moellendorff 1913, p. 148-150.

58. Ford 2002, p.93-99. Cf. Pindare, Nem. 5. 1-3, Isth. 2. 44-48. Voir aussi l'interprétation socio-poétique de Gentili 1984, p. 203-231. 
élaboration théorique de la mimesis, mais bel et bien la première formulation que nous ayons d'une confrontation entre peinture et poésie dans le cadre d'une «théorie du monument ${ }^{59}$. De leur côté, les odes de Pindare, notamment la cinquième Néméenne et la deuxième Isthmique, explicitent cette relation agonistique qu'au début $d u V^{e}$ siècle av. J.-C. entretiennent les arts visuels et ceux de la parole.

Les comparaisons entre poésie et arts plastiques chez les poètes lyriques hiérarchisent donc deux modalités distinctes de la communication commémorative en fonction des moyens de représentation propres à chacune : la parole mobile, d'un côté, la matérialité statique, de l'autre. Le point de vue d'Empédocle, qui ne pouvait pas ignorer la position des lyriques dans ce débat, est radicalement nouveau. Il consiste en l'abolition de toute forme de hiérarchie entre les arts par la création d'une catégorie unique qui recouvre toutes les activités démiurgiques $(253-257=\mathrm{B} 17.22-26)$ : aimantes et accomplissent des œuvres de jonction,

Les $\ddot{p} \theta \mu \iota \alpha$ हैp $\gamma \alpha$ qui traditionnellement reviennent à Héphaïstos ou à Athéna sont subsumés sous l'emprise de la puissance cosmique de l'Amour, dont la poésie et la peinture ne sont que deux manifestations possibles. La question des relations entre les arts plastiques et ceux de la parole quant à leurs modes et moyens de représentation bascule avec Empédocle dans le domaine de l'ontologie ${ }^{60}$. D'entrée de jeu, la Muse, déesse de la parole révélée dont l'action peut être identifiée à celle de l'Amour ${ }^{61}$, guide le char du poète (B3. 3-5) : le poème est ainsi placé sous la domination du principe cosmogonique de l'assemblage de façon à ce que son déploiement corresponde formellement avec celui des autres « œuvres de jonction » réalisées par Aphrodite artisane. De son côté, la peinture, qui constitue l'illustration privilégiée du mélange zoogonique, est également, on l'a déjà vu, une « œuvre de jonction » gouvernée par la puissance d'Amour. Du moment que l'on retrouve dans la sphère des techniques les traits immanents de

59. L'expression est de Webster 1939, p. 173.

60. Porter 2010, p. 138-169, attribue aux présocratiques le fait d'avoir posé les conditions matérielles et sensorielles pour l'émergence d'une théorie esthétique. Dans les pages qu'il consacre à Empédocle (p. 152-158), où il est question entre autres de l'analogie des peintres, le rapport entre présupposés ontologiques et formes symboliques n'est pas évoqué.

61. Cf. Ps. Hippol. Refut. 7.31.3-4 (= ad. B131). Selon Hippolyte, le discours d'Em-

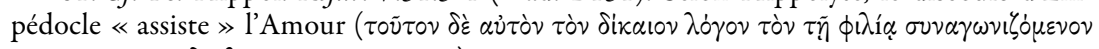

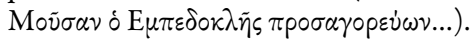


l'Amour, la doctrine reconnaît une conformité métaphysique avec la puissance cosmique dont l'une des conséquences est de rendre insignifiante toute hiérarchisation des métiers répondant à des honneurs mondains (B3. 6-8). À cet égard, il est significatif que parmi les nombreux procédés et objets techniques dont Empédocle tire des analogies ${ }^{62}$ on ne trouve pas des arts qui opèrent par extraction, comme la sculpture. À titre spéculatif, la seule hiérarchisation des arts envisageable dans la perspective cosmologique d'Empédocle serait entre les arts qui procèdent par assemblage ou mélange et ceux qui agissent par séparation : les premiers relèveraient de l'Amour et les seconds de la Haine. Dans tous les cas, chez Empédocle la poésie et les arts visuels ne se définissent pas en fonction de pratiques sociales instituées, comme dans la lyrique, mais par rapport à une puissance cosmique dont ils partagent les traits essentiels.

Image et ressemblance : le rapport à Parménide

L'écart réflexif que l'analogie des peintres d'Empédocle crée vis-à-vis du poème de Parménide est, d'un point de vue philosophique, plus fondamental et explicite que l'écart créé par rapport à la poésie lyrique. Plus fondamental parce qu'il touche, comme nous l'avons déjà annoncé, à la dimension référentielle d'un genre poétique nouveau qui se donne pour objet la totalité du réel. Le poème cosmologique offre à ses destinataires une représentation sensible de cette totalité, une comparaison qui, comme celles d'Homère, ramène l'objet du récit au champ d'expérience de l'auditoire. Dans le poème de Parménide, cette image est introduite au point culminant de la définition de l'objet du discours, à savoir l'étant est dit «sem-

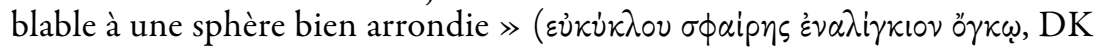
28 B8. 43). Cette comparaison n’a visiblement pas échappé à Empédocle, lequel, de son côté compare l'objet du discours, à savoir la génération du vivant à partir des quatre éléments, avec les procédés des peintres qui, à partir des pigments, « composent des figures semblables à toutes les

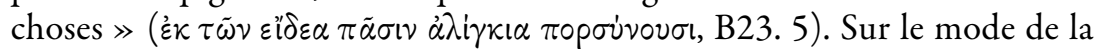
ressemblance, les deux doctrines offrent l'image de leurs objets de discours respectifs.

La première partie du poème de Parménide développe, comme on sait, les conséquences de l'interdit jeté sur le non-être en énonçant de manière systématique et argumentée l'ensemble des prédicats qui conviennent à l'être. Au cours de cette argumentation, l'être qui est l'objet du discours s'enrichit progressivement d'un certain nombre de prédicats essentiels, soumis lors de leur adoption à un examen de compatibilité avec la proposition affirmative « est ». L'étant porteur de ces prédicats est unique et 
indivisible, substantiellement immuable, immobile et n'entretient de rapports d'identité qu'avec lui-même ${ }^{63}$. Il est le produit d'un discours autoréférentiel auquel rien ne saurait correspondre dans le monde phénoménal, si ce n'est, à titre comparatif, l'image d'une sphère ${ }^{64}$ (DK 28 B8. 36-45) :

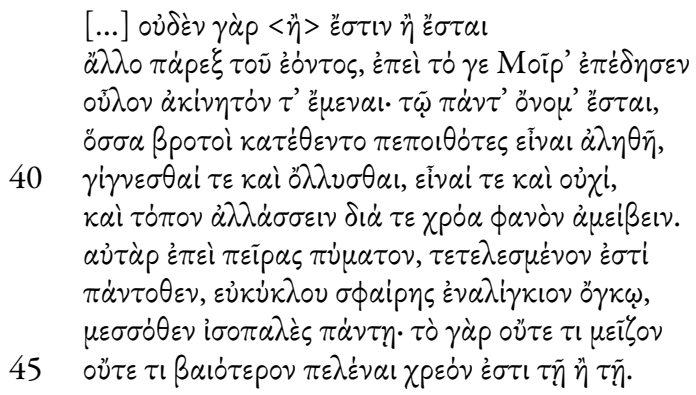

[...] Rien en effet n'est ni ne sera

D'autre à côté de ce qui est, puisque celui-ci, le Destin l'a lié

De façon à être entier et immuable; c'est pourquoi toutes seront nom,

Les choses que les mortels ont posées, persuadés qu'elles sont véridiques,

40 Naître et périr, être et n'être pas,

Échanger le lieu et modifier la brillante couleur.

Or puisque la limite est extrême, il est accompli

Partout, semblable à la masse d'une balle bien arrondie,

S'élançant, à partir du centre, avec une poussée égale partout ;

45 car il ne faut pas qu'il soit en rien plus grand ni en rien plus faible, ici ou là.

Avec cette analogie, Parménide arrive au point critique de la doctrine. La représentation de la sphère clôt ce que la déesse chargée de livrer l'enseignement qualifie comme «le récit digne de foi et la pensée autour de la

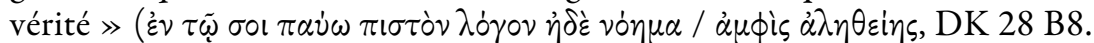
50-51). La suite du poème fait aussi partie de son enseignement, mais elle a un tout autre statut: il s'agit des «opinions des mortels » que l'on apprend, dit-elle, «en écoutant l'ordre trompeur de mes paroles » $\left(\delta \dot{\delta} \xi \alpha \varsigma \delta^{\prime}\right.$

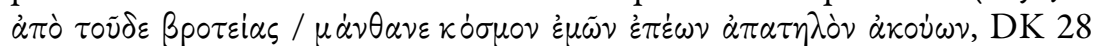
B8. 51-52). Par ces mots commence en effet un récit cosmogonique qui,

63. Cf. Bollack et Wismann 1974.

64. On distingue deux lignes d'interprétations majeures concernant la sphère de Parménide : il y a, d'une part, la tradition péripatéticienne, qui tend à identifier la sphère à un référent externe concret, et, de l'autre, un courant platonicien, qui voit dans la sphère une figure symbolique, soit de l'être en sa totalité, soit de l'une de ses propriétés. Pour un aperçu de l'ensemble des interprétations, voir Tarán 1965, p. 150 sqq. 
contrairement à la thèse de l'unicité immuable de l'être défendue dans la première partie du poème, repose sur l'introduction d'une dualité, le feu et

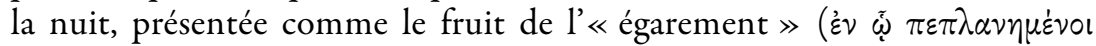

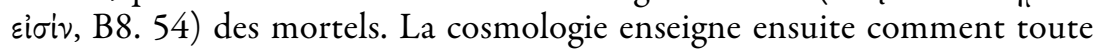
chose dans le monde résulte du mélange de ces deux entités originelles (DK 28 B12). Entre le discours «vrai » et les « opinions des mortels », l'analogie de la sphère constitue comme une frontière entre deux discours qui different et par leurs présupposés ontologiques et par leur prétention à la vérité. La sphère est à la fois le seul objet auquel on puisse comparer l'être dans l'ensemble de ses déterminations et une représentation sensible à la portée des mortels.

Le dépassement conceptuel du monisme parménidien implique chez Empédocle la suppression de deux des attributs fondamentaux de l'être : l'unité et l'immuabilité. Afin de concevoir la pluralité en mouvement sans présupposer la négativité, Empédocle postule, on l'a vu, la permanence du même au moyen de l'alternance cyclique de l'unification et de la séparation cosmiques. Ce nouveau schéma cosmologique qui permet de surmonter le monisme n'implique pas moins un déplacement considérable sur le plan des représentations. En ce sens, plusieurs éléments dans le texte de l'analogie des peintres indiquent qu'elle exprime une prise de position explicite à l'égard de l'analogie de la sphère de Parménide. Le premier indice est le choix même de la peinture comme thème de la représentation emblématique du devenir. En effet, dans les vers qui précèdent la comparaison de la sphère, Parménide énumère les modalités du changement que les mortels expriment dans leur langage courant sans se rendre compte que leur expression implique que le non-être soit : parmi elles figurent la génération et la transformation chromatique (DK 28 B8. 39-41). Ces deux modalités du changement, prises ensemble, constituent de leur côté le noyau thématique de l'analogie d'Empédocle, où le mélange des pigments entre les mains des peintres renvoie au mélange élémentaire d'où proviennent toutes les espèces vivantes. Or les composantes élémentaires des mélanges résultent à leur tour de l'éclatement de ce dieu solitaire et bienheureux auquel Empédocle, en une claire allusion à la sphère de Parménide et à la plénitude ontologique qu'elle symbolise, donne le nom de Sphairos. Les mains de la déesse (ou des peintres) prennent le relais de la sphère ; elles figurent, à partir des débris du dieu que sont les éléments, des formes mortelles qui, d'un point de vue cosmologique, ne sont jamais qu'une anticipation de la reconstitution du dieu sphérique à venir.

Un deuxième aspect à propos duquel l'analogie des peintres se situe explicitement par rapport à l'analogie de la sphère est celui de la ressemblance de l'image vis-à-vis de l'objet du discours. Les deux analogies ont recours à des adjectifs homériques parfaitement équivalents pour marquer 
la similitude entre la représentation imagée et le contenu même du dis-

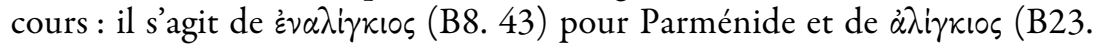
5) pour Empédocle. L'irruption dans le discours de Parménide d'une représentation sensible thématisée par un adjectif exprimant la comparaison

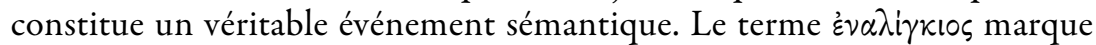
en effet le passage de l'appréhension logique de l'être à sa figuration spatiale. La limite, désignée aux vers B8. 42-43, qui exprime spatialement la complétude de l'étant constitue aussi la limite du raisonnement logique qui exprime l'identité à soi. Parménide construit au moyen de la comparaison un système de correspondances selon lequel, d'une part, le contour visible de la balle figure l'identité de l'étant, et de l'autre, sa masse expansive représente l'acte d'être $(\pi \varepsilon \lambda \dot{\varepsilon} \nu \alpha \iota)$ également partout, excluant toute différence, tout excès ou manque ${ }^{65}$. L'objet du discours de Parménide n'est figurable que sous cette forme-là, toute autre représentation évoquée dans le

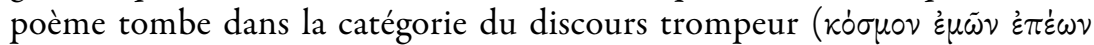
$\left.\dot{\alpha} \pi \alpha \tau \eta \lambda o^{\circ}, \mathrm{B} 8.52\right)$. C'est notamment le cas de la dualité formée par le feu et la nuit, dont le mélange produit, sous les ordres de « la divinité qui gouverne tout », le monde phénoménal (DK 28 B12). Dans tous les cas la sphère de Parménide est une icône sans comparable, si ce n'est avec l'étant, c'est-à-dire avec l'objet même du discours autoréférentiel qui l'a produite. À l'inverse de Parménide, Empédocle se donne une analogie qui contient virtuellement toutes les formes possibles dans leur devenir: les peintres composent des figures «semblables à toutes les choses » (B23. 5). La cause à l'origine de cette ressemblance est d'ordre onto-cosmologique : l'analogie des peintres ramène le rapport entre les divers contenus (peinture, v. 1-5; monde, v. 5-8; poème, v. 9-11) à une identité concrète inhérente à leur fondement (la puissance cosmique de l'Amour). La construction poétique de cette identité sur laquelle reposent les ressemblances se fait au moyen de la métaphore de la «source $\gg(\pi \eta \gamma \dot{\eta})$, introduite en guise de conclusion de l'analogie des peintres. Dans l'œuvre d'Empédocle, ce terme peut effectivement désigner à la fois l'origine élémentaire du vivant (B23. 10), l'origine de la parole poétique (B3.2) et les cinq sens à travers lesquels on saisit le monde $(\mathrm{B} 143)^{66}$. Dans l'analogie des peintres, le mot est employé à dessein pour faire converger formes symboliques (poésie et peinture) et monde phénoménal: toutes les « œuvres de jonction » découlent d'une même source d'origine divine (B23. 9-11). En d'autres termes, la source de la représentation n'est pas exogène à son objet, ce qui exclut d'emblée la

65. Je suis sur ce point les interprétations de Tarán 1965, p. 151 et de Bollack et Wismann 1974.

66. Picot 2004 élucide le réseau métaphorique de l'irrigation chez Empédocle, qui connecte la parole poétique, le monde phénoménal et les cinq sens. 
possibilité de la tromperie $(\dot{\alpha} \pi \dot{\alpha} \tau \eta)$, un autre trait parménidien par rapport auquel Empédocle se situe.

Ainsi, plutôt que d'un rapport d'analogie ou de correspondance entre différentes sphères de représentation - cosmique et humaine, physiologique et artistique, poétique et picturale -, on devrait parler dans le cas d'Empédocle d'un rapport d'identité qui s'exprime de diverses manières ${ }^{67}$. Plus précisément, l'analogie n'est pas fondée ici sur un transfert sémantique comme celui que Parménide construit à propos de la sphère, entre l'appréhension logique et la représentation, au contraire, ce qui fonde l'analogie empédocléenne est l'identité principielle qui sous-tend la catégorie $\mathrm{d}^{\prime}$ « œuvre de jonction ». Toute ressemblance dans le comportement des choses ou des processus est pour Empédocle la preuve pour ainsi dire palpable qu'ils doivent comporter quelque chose de commun. Dans un univers clos de présupposés ontologiques, l'analogie est autant une illustration qu'une démonstration.

\section{L'analogie : entre la tradition et l'auvre individuelle}

J'ai voulu dans cette étude révéler certains aspects essentiels d'une doctrine cosmologique que son auteur exprime au détour d'une analogie. En explorant la dimension paradigmatique du fait artisanal, on a pu constater qu'Empédocle mobilise un système d'images qui d'une certaine manière le dépasse. Les représentations de la technique dans la poésie archaïque appartiennent en effet à une tradition qui, comme on sait, remonte à la poétique indo-européenne. En même temps, ce vecteur thématique, en particulier l'analogie de la peinture au fragment B23, fait ressortir la singularité d'Empédocle. L'analyse de ce passage a permis de repérer certains points d'inflexion, des discontinuités et des déplacements de sens historiquement déterminés qui pour nous, modernes, ont tendance à passer inaperçus sous

67. Kamtekar 2009 propose à partir de B109 une interprétation non substantialiste de la connaissance chez Empédocle. Selon l'auteure, dans la doctrine d'Empédocle la pensée et les représentations n'auraient pas la consistance élémentaire qu'Aristote (De anima, I. 2 et 5) et Théophraste (Sens. 1-2,10) leur prêtent. Kamtekar attribue au contraire à Empédocle une pensée capable de schématiser les contenus des diverses représentations : « in this process of knowledge acquisition, it is their representational content (not their material composition) which is significant $\gg($ p. 235). Cela suppose dans une certaine mesure qu'Empédocle ait résolu le problème philosophique du rapport du sujet et de l'objet. Outre le fait que l'on n'a pas d'éléments concrets pour conclure à une désubstantialisation des représentations chez Empédocle, Kamtekar contourne dans son interprétation le problème majeur qui se pose à Empédocle - et à tous les penseurs pré-modernes - à savoir celui de trouver le principe de sa propre pensée dans ce qu'il pense, en d'autres termes: comment l'activité de penser peut correspondre à l'objet pensé. Pour Empédocle, l'équivalent du sujet qui pense doit être trouvé dans l'objet qui est une totalité ; l'instance du sujet, de la res cogitans, n'existe pas, ou bien c'est le cosmos lui-même. On trouve certes chez Empédocle les traces de cette aporie, mais il ne la résout pas. 
l'expression métaphorique. À plus d'un égard, l'étude des métaphores et des analogies chez les philosophes présocratiques éclaire d'une lumière nouvelle la dialectique de l'œuvre individuelle et de la tradition. Je ne peux m'empêcher de citer pour conclure un passage de Hans Blumenberg, un auteur qui a fait de la métaphorologie une branche à part entière de l'histoire de la philosophie où, de manière presque aphoristique, il élabore le type même de recherche que j'ai souhaité mettre en œuvre ici :

On trouve chez chaque penseur des métaphores qui semblent plus appartenir à son époque qu’à lui-même. Elles permettent parfois de jeter un regard sur l'arrière-plan de ses questions et de ses décisions sur les choses. Quand elles le font entrer dans l'horizon de son époque - et le montrent éventuellement comme un être livré à l'esprit du temps -, ces métaphores restent toutefois instructives par les déplacements et les déformations qu'on peut encore leur faire subir et qui permettent d'appréhender la force d'une individualité face à la généralité ${ }^{68}$. 


\section{BIBLIOGRAPHIE}

ARnim, H. von 1902: «Die Weltperioden bei Empedokles », dans Festschrift Theodor Gomperz dargebracht zum siebzigsten Geburtstage am 29. März 1902, von Schülern, Freunden, Collegen, Vienne, p. 16-27.

Bignone, E. 1916: I Poeti filosofi della Grecia. Empedocle, studio critico, traduzione e commento delle testimonianze e dei frammenti, Turin, 1916 (Il Pensiero greco, 11).

BOLlaCK, J. 1965-1969 : Empédocle, 3 vol., Paris, 1965-1969 (Le Sens commun). [Réimpr. Paris, 1992, 3 vol., Collection Tel, 201-203.]

— \& H. Wismann 1974 : «Le moment théorique (Parménide, fr. 8, 42-49) », Revue des sciences humaines, 154 (1974), p. 203-212.

Blumenberg, H. 1990 : Le Souci traverse le fleuve, Paris, 1990 (trad. par O. Mannoni de Die Sorge geht über den Fluss, Francfort/Main, 1988).

- 2001 : Ästhetische und metaphorologische Schriften, sélection et postface d'A. Haverkamp, Francfort/Main, 2001 (Suhrkamp-Taschenbuch Wissenschaft, 1513).

BRUnO, V. J. 1977 : Form and Colour in Greek Painting, Londres, 1977.

CALAME, C. 1996 : L'Éros dans la Grèce antique, Paris, 1996 (L'Antiquité au présent).

Chantraine, 1948 : Grammaire homérique. Tome I, Phonétique et morphologie, Paris, $1948\left[{ }^{1} 1942\right]$ (Collection de philologie classique, 1).

Croisille, J.-M. 1985 : Pline l'ancien, Histoire naturelle, Livre XXXV, Texte établi et traduit par —, Paris, 1985 (Collection des Universités de France).

Detienne, M. et Vernant, J.-P. 1974 : Les Ruses de lintelligence. La Mètis des Grecs, Paris, 1974 (Nouvelle bibliothèque scientifique).

Diels, H. 1901 : Poetarum philosophorum fragmenta, Berlin, 1901 (Poetarum Graecorum fragmenta, III/1).

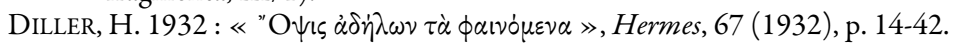

FORD, A. 2002: The Origins of Criticism. Literary Culture and Poetic Theory in Classical Greece, Princeton, NJ, 2002.

FRÄNKEL, H. 1921 : Die homerischen Gleichnisse, Gottingue, 1921.

Gallavotti, C. 1975 : Empedocle, Poema fisico e lustrale, Milan, 1975.

Graham, D. W. 1988 : « Symmetry in the Empedoclean Cycle », Classical Quarterly, n.s. 38 (1988), p. 297-312.

Gentili, B. 1984 : Poesia e pubblico nella Grecia antica: da Omero al V secolo, Rome-Bari, 1984.

HÖLSCHER, U. 1965 : « Weltzeiten und Lebenszyklus. Eine Nachprüfung der EmpedoklesDoxographie », Hermes, 93 (1965), p. 7-33.

Ierodiakonou, K. 2004 : «Empedocles and the Ancient Painters », dans L. Cleland \& K. Steras (éd.), Colour in the Ancient Mediterranean World, Oxford, 2004 (BAR. International series, 1267), p. 91-95.

- 2005: «Empedocles on Colour and Colour Vision », Oxford Studies in Ancient Philosophy, 29 (2005), p. 1-37.

Inwood, B. 2001 : The Poem of Empedocles. A Text and Translation with an Introduction, $2^{\mathrm{c}}$ éd., Toronto, 2001 [ $\left.{ }^{1} 1992\right]$ (Phoenix. Supplementary volume $39=$ The Phoenix Presocratics, 3).

JANKO, R. 2004 : « Empedocles, On Nature I 233-364: a New Reconstruction of $P$. Strasb. gr. Inv. 1665-6 », Zeitschrift für Papyrologie und Epigraphik, 150 (2004), p. 1-26.

- 2005: «Empedocles' Physica Book 1: A new Reconstruction », dans A. L. Pierris (éd.), The Empedoclean Kóruos: Structure, Process and the Question of Cyclicity, Proceedings of the Symposium Philosophiae Antiquae Tertium Myconense July $6^{\text {th }}$-July 13 ${ }^{\text {th }}$, 2003. Part 1: Papers, Patras, 2005 (Conference Series, 3), p. 93-135. 
Kamtekar, R. 2009 : « Knowing by Likeness in Empedocles », Phronesis, 54 (2009), p. 215-238.

KINGSLEY, P. 2003 : Reality, Inverness (CA), 2003.

KRANZ, W. 1938 : « Gleichnis und Vergleich in der frühgriechischen Philosophie », Hermes, 73 (1938), p. 99-122.

LanatA, G. 1963 : Poetica preplatonica: Testimonianze e frammenti. Texte, traduction et commentaire, Florence, 1963 (Biblioteca di Studi superiori, 43).

LAKS, A. 2013 : « Sur quelques modalités de la raison pratique dans les cosmo-ontologies présocratiques », dans G. Rossi (éd.), Nature, Reason and Normativity in Ancient Philosophy, Hildesheim (à paraître).

LLOYD, G. E. R. 1966 : Polarity and Analogy. Two Types of Argumentation in Early Greek Thought, Cambridge, 1966.

LONG, A. A. 1974 : «Empedocles' Cosmic Cycle in the 'Sixties », dans A. P. D. Mourelatos (éd.), The Pre-Socratics. A Collection of Critical Essays, Garden City, NY (Modern Studies in Philosophy), p. 397-425.

LUCAS, W. D. 1968 : Aristotle, Poetics. Introduction, commentary and appendixes, Oxford, 1968.

Mansfeld, J. \& PRIMAVESI, O (éd.) 2011 : Die Vorsokratiker, Griechisch/Deutsch. Ausgewählt, übersetzt und erläutert, Stuttgart, 2011 (Reclam Bibliothek).

Martin, A. \& O. PRIMAVESI 1999: L'Empédocle de Strasbourg (P. Strasb. gr. Inv. 16651666). Introduction, édition et commentaire, Berlin/New York, 1999.

Monro, D. B. 1891 : A Grammar of the Homeric Dialect, Oxford, ${ }^{2} 1891$.

MourelatoS, A. P. D. 1987 : «Quality, Structure, and Emergence in Later Pre-Socratic Philosophy », in J. J. Cleary (éd.), Proceedings of the Boston Area Colloquium in Ancient Philosophy, 2, Lanham (Md), 1987, p. 127-194.

NÜNLIST, R. 1998 : Poetologische Bildersprache in der frühgriechischen Dichtung, Stuttgart, 1998 (Beiträge zur Altertumskunde, 101).

O'Brien, D. 1969 : Empedocles'Cosmic Cycle. A Reconstruction from the Fragments and Secondary Sources, Cambridge, 1969 (Cambridge Classical Studies).

- 1970 : « The Effect of a Simile: Empedocles' Theories of Seeing and Breathing », The Journal of Hellenic Studies, 90 (1970), p. 140-179.

Picot, J.-C. 2004 : « Les Cinq Sources dont parle Empédocle », Revue des études grecques, 117 (2004), p. 393-446; corrigenda : 118 (2005), p. 322-325.

PANZerbieter, F. 1844 : Beiträge zur Kritik und Erklärung des Empedokles, Meiningen, 1844.

PORTER, J. I. 2010 : The Origins of Aesthetic Thought in Ancient Greece: Matter, Sensation and Experience, Cambridge, 2010.

PRIMAVESI, O. 2008 : Empedokles, Physika I. Eine Rekonstruktion des zentralen Gedankengangs, Berlin/New York, 2008 (Archiv für Papyrusforschung und verwandte Gebiete, Beiheft 22).

Rashed, M. 2007 : «The Structure of the Eye and Its Cosmological Function in Empedocles: Reconstruction of Fragment 84 D.-K. », dans S. Stern-Gillet et K. Corrigan (éd), Reading Ancient Texts (I): Presocratics and Plato. Essays in Honour of Denis O'Brien, Leyde-Boston (Brill's studies in intellectual history, 161), p. 21-39.

Rouse, W. H. D. 1902 : Greek Votive Offerings: An Essay in the History of Greek Religion, Cambridge, 1902.

SCHEIBLER, I. 1974: «Die 'Vier Farben' der griechischen Malerei », Antike Kunst, 17 (1974), p. 92-102.

SEDLEY, D. 2007 : Creationism and its Critics in Antiquity, Berkeley, 2007 (Sather classical lectures, 66). 
SKARSOULI, P. 2009 : « S'interroger sur la relation entre couleurs et mots. Le terme phármakon chez Empédocle », dans M. Carastro (éd.), L'Antiquité en couleurs : catégories, pratiques, représentations, Grenoble (Horos), p. 165-177.

Solmsen, F. 1965: «Love and Strife in Empedocles' Cosmology », Phronesis, 10 (1965), p. 109-148.

TARÁN, L. 1965 : Parmenides, a text with translation, commentary and critical essays, Princeton, 1965.

THAYER, H. S. 1975 : « Plato's Quarrel with Poetry: Simonides », Journal of the History of Ideas, 36 (1975), p. 3-26.

TRÉPANIER, S. 2003 : «Empedocles on the Ultimate Symmetry of the World », Oxford Studies in Ancient Philosophy, 24 (2003), p. 1-57.

Warren, J. 2007 : Presocratics: Natural Philosophers before Socrates, Berkeley, 2007 (Ancient philosophies, 2).

WebsteR, T.B. L. 1939: «Greek Theories of Art and Literature Down to 400 B.C. », Classical Quarterly, 33 (1939), p. 166-179.

Wersinger, A.-G. 2008 : La Sphère et l'intervalle. Le schème de l'harmonie dans la pensée des anciens Grecs d'Homere à Platon, Grenoble, 2008 (Horos).

Wright, M. R. 1981 : Empedocles. The Extant Fragments, ed. with an introd., comm. \& concordance, New Haven, 1981.

WilAMOWITZ-MOELLENDORFF, U. von 1913 : Sappho und Simonides. Untersuchungen über griechische Lyriker, Berlin, 1913.

ZELLER, E. 1920 : Die Philosophie der Griechen in ibrer geschichtlichen Entwicklung, II 1, 2, éd. par W. Nestle, Leipzig, ${ }^{6} 1920$. 